\title{
Applications of Pulsed Light Decontamination Technology in Food Processing: An Overview
}

\author{
Ronit Mandal ${ }^{1}\left(\mathbb{D}\right.$, Xanyar Mohammadi $^{1}$, Artur Wiktor ${ }^{2} \oplus$, Anika Singh ${ }^{1}$ and \\ Anubhav Pratap Singh 1,*(1) \\ 1 Food, Nutrition, and Health, Faculty of Land \& Food Systems, 2205 East Mall, University of British \\ Columbia, Vancouver, BC V6T 1Z4, Canada; ronit.mandal@ubc.ca (R.M.); xanyar91@mail.ubc.ca (X.M.); \\ anika.singh@ubc.ca (A.S.) \\ 2 Department of Food Engineering and Process Management, Institute of Food Sciences, \\ Warsaw University of Life Sciences (WULS-SGGW), 02-787 Warszawa, Poland; artur_wiktor@sggw.pl \\ * Correspondence: anubhav.singh@ubc.ca; Tel.: +1-604-822-5944
}

Received: 1 May 2020; Accepted: 19 May 2020; Published: 22 May 2020

Featured Application: This review discusses recent developments in the field of pulsed light processing. Various aspects of recent PL applications are discussed. This review shall form the basis of future research and development in the field.

\begin{abstract}
Consumers of the 21st century tend to be more aware and demand safe as well as nutritionally balanced food. Unfortunately, conventional thermal processing makes food safe at the cost of hampering nutritional value. The food industry is trying to develop non-thermal processes for food preservation. Pulsed light (PL) is one such emerging non-thermal food processing method that can decontaminate food products or food contact surfaces using white light. Exposure to intense light pulses (in infrared, visible, and ultraviolet (UV) regions) causes the death of microbial cells, rendering the food safe at room temperature. PL technology is an excellent and rapid method of disinfection of product surfaces and is increasingly being used for food surfaces and packaging decontamination, enabling the minimal processing of food. This paper aims to give an overview of the latest trends in pulsed light research, discuss principles of pulse generation, and review applications of various PL systems for the inactivation of microorganisms in vitro, in various food products, and on food contact surfaces. Effects of PL on food quality, challenges of the process, and its prospects are presented.
\end{abstract}

Keywords: pulsed light technology; fluence; food processing; food decontamination

\section{Introduction}

Currently, there is a growing concern for nutrition and food safety. Modern technology and access to the internet has made the 21st-century consumer more aware and demanding than ever before. They want safe, sound, and healthy foods of good quality, having an adequate supply of required nutrients, but devoid of any preservatives [1]. Conventionally, the food industry has used preservatives or thermal processing for the extension of shelf-life.

The deleterious effect of heat on the nutritional attributes and sensory qualities of food is now common knowledge [2,3], and the food industry is experiencing a paradigm shift towards minimal and non-thermal technologies. This has prompted food processing researchers to develop various technologies like ohmic heating, radiofrequency (RF), microwave (MW), and infrared (IR) heating, high pressure processing (HPP), ultrasound (US), pulsed electric field (PEF), pulsed light (PL), cold plasma $(\mathrm{CP})$, oscillating magnetic field (OMF) etc. as alternates to the conventional thermal process. These technologies have the potential to gradually replace the thermal methods practiced by the food 
industry, especially because they are carried out at ambient and sub-lethal temperatures; and in this way are disposing of the antagonistic impacts of thermal technologies. Each of these novel technologies constitutes an altogether separate field of studies themselves. However, the purpose of this literature review is to study and intimate the readers regarding the critical fundamentals and applications of pulsed light (PL) technology in food processing.

PL treatment generally consists of applying a series of high intensity, short bursts of light pulses to kill microorganisms. PL is synonymous with the terms pulsed ultraviolet light (PUV), intense light pulse (ILP), high-intensity broad-spectrum pulsed light (BSPL), high-intensity pulsed UV light (HIPL), or pulsed white light (PWL) $[4,5]$. PL has a great potential to enhance the keeping quality of food products [6,7] by destroying microorganisms such as Listeria monocytogenes within a few seconds [1]. PL was successful in reducing the total microbial load in vitro when inoculated on the surface of agar plates [1]. So, it can be claimed to have the potential to preserve food materials. Also, PL technology can be effectively used to sterilize packaging materials [8], and equipment surfaces [9,10]. However, PL technology has its limitations to be used for food applications due to the opacity of food products and their non-uniform surfaces, and possible temperature rise (due to the effects of the infrared region) leading to deterioration in organoleptic qualities.

In 1996, the U.S. Food and Drug Administration (FDA) approved the PL technology applications 'for the production, processing and handling of foods' and decontaminating food contact surfaces [11]. They recommended the use of PL treatment using xenon lamp having surface emission of wavelengths ( $\lambda$ ) between 200 and $1100 \mathrm{~nm}$, with the cumulative treatment not more than $12 \mathrm{~J} / \mathrm{cm}^{2}$ and pulse width not greater than $2 \mathrm{~ms}$ [4]. Since then, a considerable increase in research and development in the field of PL technology in food industry has taken place. As a result, more than 250 articles, including reviews, patents, and chapters have been published. Figure 1 shows a steady rise in publications after the year 2000. Numerous PL systems and configurations have been manufactured. Successful intervention for decontaminating fresh-cut fruits and vegetables, meat surfaces, equipment-contact surfaces, and clear liquid foods. Efforts are being made to extend it to turbid and opaque liquid foods, seeds, powders, ready-to-eat meals, and other thermally labile food commodities. With the growing demand for safe and nutrient-rich foods, thorough research should make the process ready to be adopted by the food industry sooner or later.

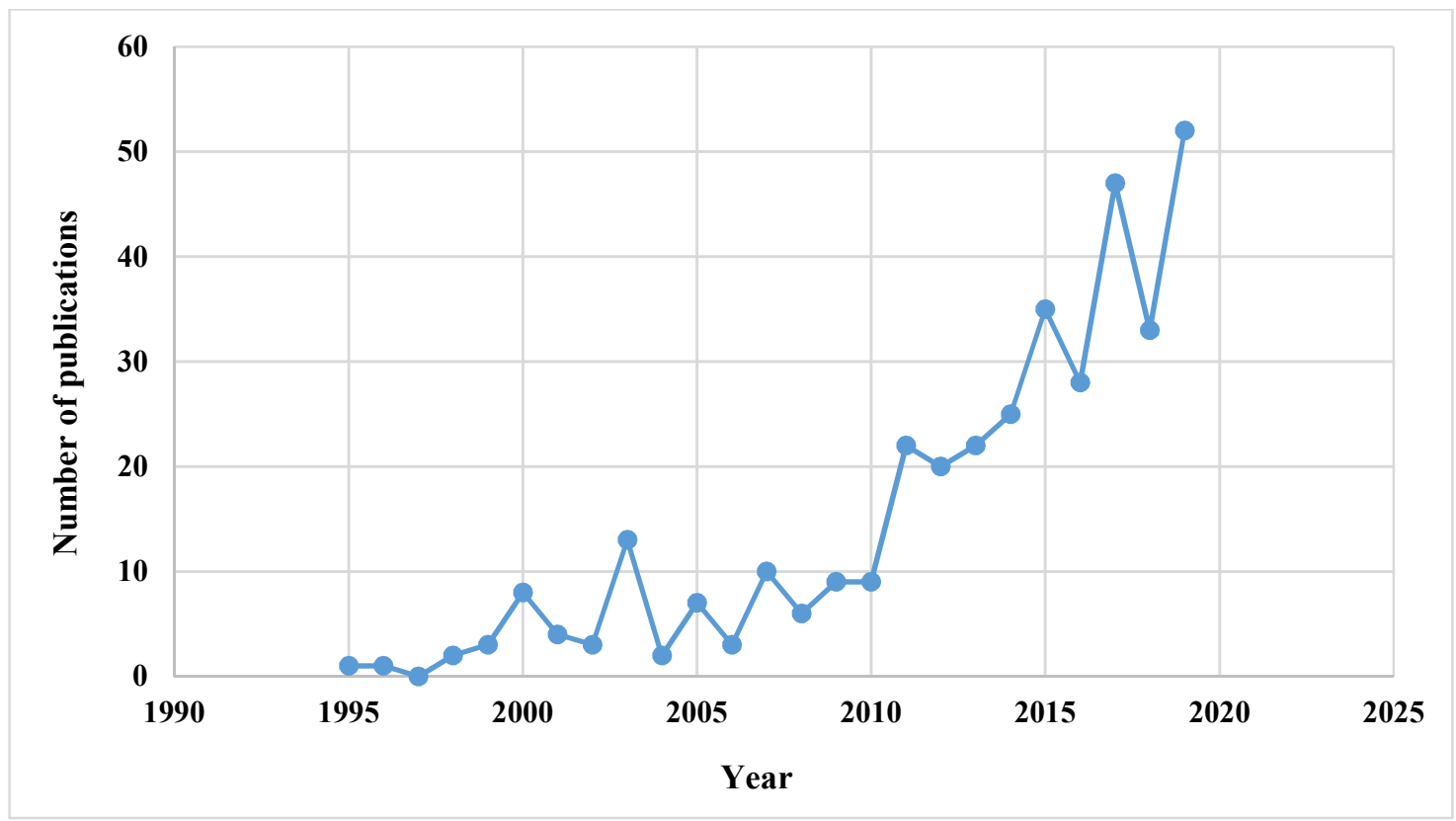

Figure 1. Increase in the number of publications in the field of pulsed light. 
Thus, the main objective of this paper is to review the recent developments of PL processes and applications that have been in practice. A systematic literature search has been conducted in the database (Web of Science, Clavariate Analytics, United States) for literature obtained from keywords like pulsed light and pulsed UV light. Web of Science was selected as it has huge collection of articles and citations with many journals indexed, with a very user-friendly interface. The search criteria adopted by authors are given in Figure 2. It is apparent that there have been many reviews earlier about PL technology, some of which are considered in our analysis. The Table 1 discusses the particulars of some of those reviews conducted earlier. Based on these reviews, we have updated the state of literature concerning the latest trends in PL processing. Processing systems that have been developed have been summarized. The detailed inactivation mechanisms of microorganisms by using the PL technology are also discussed within the sections. Further, the different PL applications in the food industry, effects on food quality, future scope, and challenges are highlighted.

\section{Search criteria}

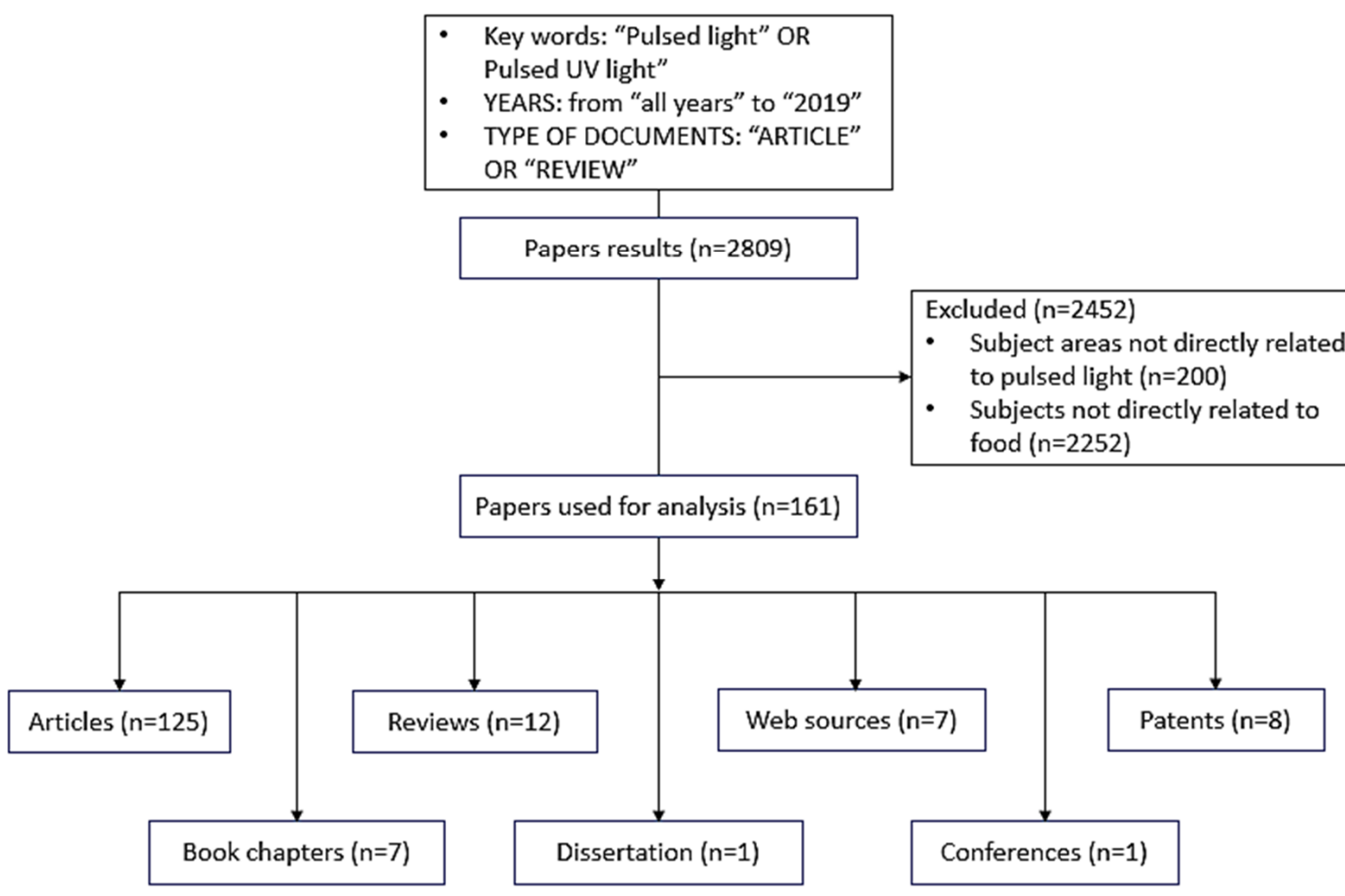

Figure 2. Search criteria for conduction of literature search with keywords "pulsed light", "pulsed UV light" and excluding results not related to the food applications. For the review, 161 articles were referred. 
Table 1. Previously published review papers on PL processing.

\begin{tabular}{|c|c|}
\hline Paper Title, Authors and Year of Publication & Key Contents \\
\hline Pulsed light technology; Barbosa-Canovas et al.; 2000 & $\begin{array}{l}\text { - Systems developed for PL processing, } \\
\text { - } \quad \text { Possible bacterial inactivation mechanisms, } \\
\text { - Critical factors affecting the PL process efficacy, } \\
\text { - } \quad \text { Results from industrial sources, } \\
\text { - } \quad \text { Research needs }\end{array}$ \\
\hline $\begin{array}{l}\text { Pulsed light for food decontamination: a review; } \\
\text { Gómez-López et a.; } 2007\end{array}$ & $\begin{array}{l}\text { - Terminologies related to PL, } \\
\text { - } \text { Microbial inactivation mechanisms, } \\
\text { - } \text { Factors affecting the inactivation, } \\
\text { - } \quad \text { Eecontamination of food, } \\
\text { Effects on nutritional and toxicological aspects }\end{array}$ \\
\hline $\begin{array}{l}\text { Pulsed-light system as a novel food decontamination } \\
\text { technology: a review; Elmnasser et al.; } 2007\end{array}$ & $\begin{array}{l}\text { - } \quad \text { Principles of PL, } \\
\text { - } \quad \text { Microbial inactivation in vitro, } \\
\text { - } \quad \text { Dain limitations, } \\
\text { Decontamination of several food products }\end{array}$ \\
\hline $\begin{array}{l}\text { Pulsed Light Treatments for Food Preservation. A Review; } \\
\text { Oms-Oliu et al.; } 2008\end{array}$ & $\begin{array}{l}\text { - } \quad \text { Principles of PL, } \\
\text { - } \quad \text { Microbial inactivation in vitro, } \\
\text { - } \quad \text { Critical process factors, } \\
\text { - } \quad \text { Microbial inactivation on packaging materials } \\
\text { and food contact surfaces, } \\
\text { - Limitations }\end{array}$ \\
\hline $\begin{array}{l}\text { The Sensory Quality of Meat, Game, Poultry, Seafood and Meat } \\
\text { Products as Affected by Intense Light Pulses: A Systematic } \\
\text { Review; Tomasevic and Rajkovic; } 2015\end{array}$ & $\begin{array}{l}\text { - Effect of PL on the sensory quality of } 16 \text { varieties } \\
\text { of meat, meat products, game, poultry, seafood, } \\
\text { - Effect on color and odor of meat }\end{array}$ \\
\hline $\begin{array}{l}\text { Pulsed Light Treatment of Different Food Types with a Special } \\
\text { Focus on Meat: A Critical Review; Heinrich et al.; } 2016\end{array}$ & $\begin{array}{l}\text { - Principles of PL processing, } \\
\text { - } \quad \text { Efficacy of processing unpackaged and } \\
\text { packaged meat products, } \\
\text { - Decontamination of meat cutting, } \\
\text { packaging surfaces, } \\
\text { - Industrial implementation needs }\end{array}$ \\
\hline $\begin{array}{l}\text { Pulsed light processing of foods for microbial safety; Bhavya } \\
\text { and Hebbar; } 2017\end{array}$ & $\begin{array}{l}\text { - } \quad \text { Principles of PL processing, } \\
\text { - } \quad \text { PL processing systems, } \\
\text { - } \quad \text { Pode of action on microorganisms, } \\
\text { - } \quad \text { Combination of PL, with other } \\
\text { processing technologies }\end{array}$ \\
\hline
\end{tabular}

\section{Overview of PL Technology}

PL technology involves the application of intense light in the form of short pulses on a target of interest (food or food contact surface or illumination through a fluid food matrix) to destroy bacteria, yeasts, molds, and viruses present therein. The PL is nothing but ordinary white light like sunlight, except having a very high intensity, and applied over a very short period of time. PL has an electromagnetic spectrum similar to that of sunlight, ranging from ultraviolet (UV) to near infrared (IR) wavelengths [12]. This range of electromagnetic radiation includes $U V$ rays $(\lambda=200-400 \mathrm{~nm}$; UV is again classified into UV-C: $200-280 \mathrm{~nm}, \mathrm{UV}-\mathrm{B}: 280-315 \mathrm{~nm}, \mathrm{UV}-\mathrm{A}: 315-400 \mathrm{~nm}$ ), visible light $(\lambda=400-700 \mathrm{~nm})$, and infrared (IR) rays $(\lambda=700-1100 \mathrm{~nm})[4,13]$. However, the spectral output of PL is rich in UV regions, unlike solar spectrum, which is rich in visible regions [14] (p. 236). The generation of light pulses is carried out by the excitation of inert gases, like xenon in flashlamps, and the collision of gaseous molecules due to electrical pulse application. The light energy is then released in the form of 
short-duration light bursts in a highly concentrated manner (lasting for a few hundred microseconds, usually $1 \mu \mathrm{s}$ to $0.1 \mathrm{~s})[13,15]$. The light pulses produced are short-lived, but are of high intensity, summing up to around twenty thousand times the sunlight intensity at the sea level $[1,6,13]$.

Broad-spectrum pulsed light inactivates microorganisms by a combination of photo-chemical, photo-thermal, and photo-physical effects [16,17], which are discussed later. Mainly, the lethal effect is owed to the photo-chemical effect whereby light photons of the UV-C wavelengths, notably $253.7 \mathrm{~nm}$, are absorbed by the microbial DNA. Thus, it shows antimicrobial effects alike continuous UV processing [18]. Thereby, similar to continuous UV processing, rules and terms associated with photophysics and photochemistry laws have been applied for PL treatment characterization since its inception. Light-based processes are mainly quantified in terms of the cumulative energy dose imparted during treatment so that the results of PL can be uniformly reported. Typically, the term fluence is used to quantify the radiant energy for treatment. Fluence $\left(\mathrm{F}_{\mathrm{o}}\right)$ is the cumulative radiant energy comprised of all wavelengths per unit area coming from all directions incident on an imaginary infinitesimal sphere with cross-section area $\mathrm{dA}$ (unit: $\mathrm{J} / \mathrm{cm}^{2}$ ) (Figure 3).

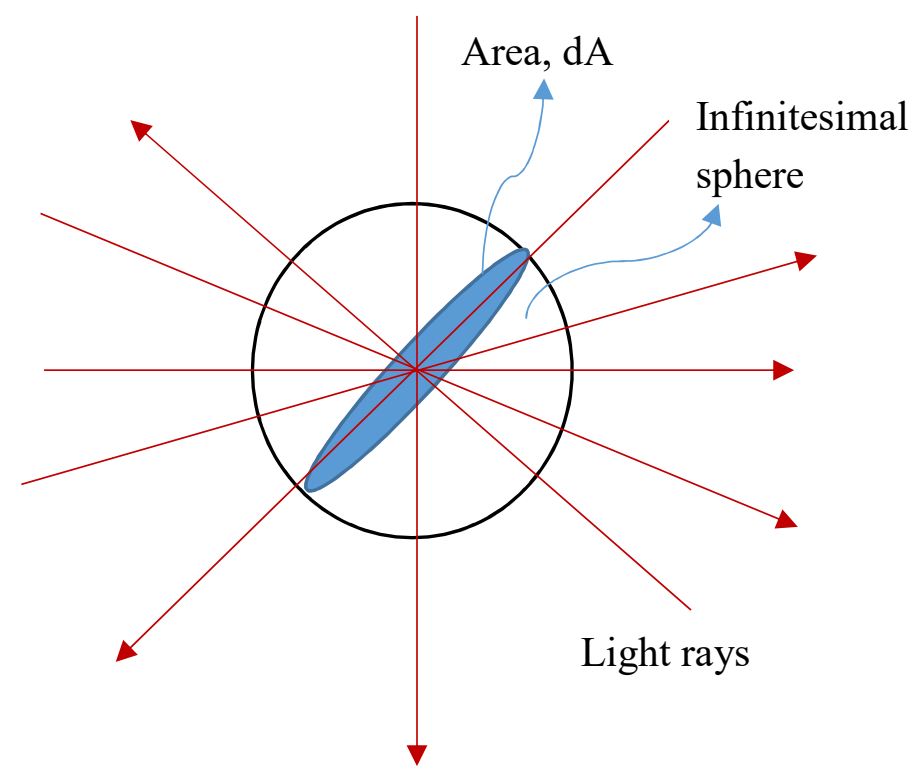

Figure 3. Graphical interpretation of fluence. Total cumulative radiant energy incident on the small sphere from all directions per unit cross-section area of the sphere.

The PL inactivation efficacy is dependent upon the delivered fluence [4]. Parameters affecting the fluence (at a particular distance from the lamp) are the pulse width $(\tau)$, treatment time $(t)$, number of pulses (n), pulse repetition rate (f) [19]. The fluence is mathematically calculated as the product of energy of each pulse, total number of pulses, and pulse frequency as expressed by Equation (1) [20].

$$
F_{o}=e \times t \times f
$$

where $F_{o}=$ fluence $\left(\mathrm{J} / \mathrm{cm}^{2}\right), e=$ energy of one pulse $\left(\mathrm{J} / \mathrm{cm}^{2}\right.$ per pulse), $t=$ treatment time $(\mathrm{s})$, and $f=$ pulse frequency $(\mathrm{Hz})$.

Other terms related to PL like fluence rate $\left(\mathrm{W} / \mathrm{cm}^{2}\right)$, spectral irradiance $\left(\mathrm{W} /\left(\mathrm{cm}^{2}-\mathrm{nm}\right)\right)$, radiance exposure $\left(\mathrm{J} / \mathrm{cm}^{2}\right)$ etc. have been described by Gómez-López and Bolton [19]. The researchers tried to introduce the fundamentals of photochemistry and biodosimetry as applicable to continuous UV light wave processing to PL tests.

Light energy, as continuous UV, has been used for a long time for disinfection and decontamination in hospitals, laboratories, etc. However, PL application involves a slightly different approach. During PL application, the electrical energy is first concentrated over a long period of time, and then discharged 
in the form of short-duration light pulses. These light flashes have more decontamination efficacy than continuous UV light application due to their higher intensity. This may arguably be due to the shorter pulse duration leading to higher impulsive power at equivalent radiant energy. This has been explained to be similar to the concept of 'impulsive force', wherein a short-duration impact force (say a hammer) produces greater impact. Pulsed UV systems can dissipate $~ 35 \mathrm{MW}$ of impulsive power for a fluence level of $3 \mathrm{~J} / \mathrm{cm}^{2}$ [21]. On the contrary, a continuous wave UV system dissipates power in the range of $0.1-1 \mathrm{~kW}$ [22]. PL treatment is proven to be more efficient in reducing pathogens like Escherichia coli O157:H7 and L. monocytogenes than continuous UV light irradiation [23]. In a recent study done by Zou et al. [24], results show E. coli inactivation by using pulsed UV-LED irradiation is more effective than continuous UV. By applying the same dose, the inactivation rate of E. coli significantly rose as the duty cycle reduced from $100 \%$ to $5 \%$ [24]. In addition to a higher decontamination power, high intensity light pulses also penetrate a larger distance through materials than continuous UV light, although both might have the same penetration depth (the distance travelled by the light before its' intensity becomes $1 /$ e of itself) $[25,26]$. Although it has been debated whether pulsed light has greater penetration depth than continuous UV, a higher effective penetration depth can be supported by the conjecture that the penetration of light waves also depends on wavelength, i.e., penetration depth of light in a substance increases as the wavelength of light decreases. As the PL includes wavelength from 200 to $1100 \mathrm{~nm}$, the lower bound wavelengths of around $200 \mathrm{~nm}$ have higher penetration depth than continuous wave UV (considering $253.7 \mathrm{~nm}$ ).

There is some heating attributed to the use of PL, which is beneficial as it may add to the decontamination process. However, it has created problems for PL application on fresh produces based on the degradation of sensory qualities [27]. Mathematically, the interaction of PL with matter can be studied by Lambert-Beer law. When light radiation of energy intensity $\mathrm{I}_{0}$ falls on a product superficially, it gets transmitted into its depth and then gets absorbed by the layers of product [4] as in Figure 4.

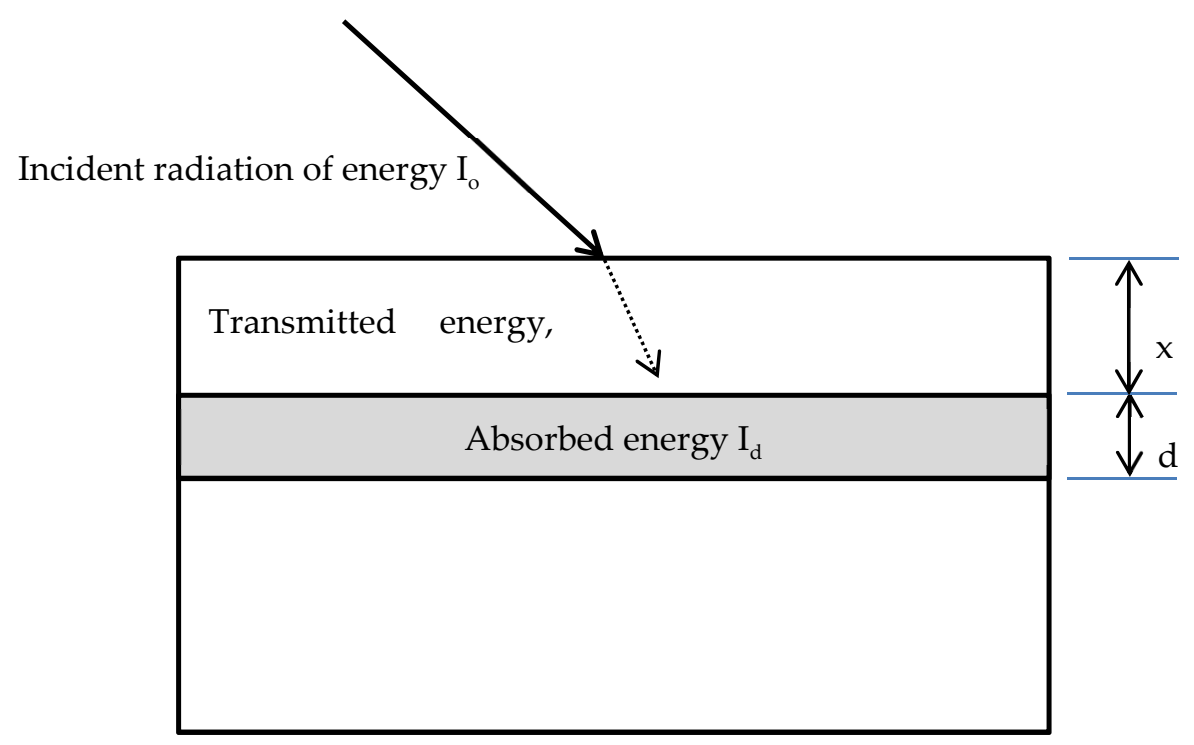

Figure 4. Interaction of light with product. When light falls on a body, part of it reflects, part of it gets absorbed and part of it transmitted into the inner layers.

The light fraction having energy I $(x)$ is transmitted as per Lambert-Beer's law (Equation (2)) as a function of the distance ' $x$ ' under the product surface, the wavelength of light, and the material extinction (absorption) coefficient. Most food materials show a decrease in light intensity while penetrating their bulk [4], which appears in the form of heat, leading to a temperature rise, given by 
Equation (3). The extinction coefficient, $\alpha$ in Equation (2), is related to the transparency or opacity of the material.

$$
\begin{gathered}
I(x)=I_{o} e^{-[\alpha] x} \\
T=\frac{I_{d}}{\rho C_{p} A d} \\
I_{d}=I(x)\left(1-e^{-[\alpha] d}\right)
\end{gathered}
$$

where $\alpha=$ extinction coefficient $\left(\mathrm{cm}^{-1}\right), \rho=$ density of body $\left(\mathrm{kg} / \mathrm{m}^{3}\right)$, and $C_{\mathrm{p}}=$ specific heat of the body $(\mathrm{J} / \mathrm{kg}-\mathrm{K})$ and $\mathrm{A}=$ surface area of body $\left(\mathrm{m}^{2}\right) . \mathrm{I}_{\mathrm{d}}$ is the absorbed energy (from the transmitted light) between the depth $\mathrm{x}$ and $\mathrm{x}+d$, given by:

$$
\frac{\mathrm{d} T}{\mathrm{~d} d}=\frac{I_{d}}{k A}
$$

Such a temperature rise creates a temperature gradient $\left(\frac{\mathrm{d} T}{\mathrm{~d} d}\right)$ (Equation (5) between the surface and the internal layers of the product, setting up conductive heat transfer $(\mathrm{k}=$ thermal conductivity of the material, $\mathrm{W} / \mathrm{m}-\mathrm{K}$ ) within the material. This dissipated heat may be utilized for the decontamination of food products [4]. However, it must be emphasized that this heating is not the primary mechanism of PL action on microbes (discussed in Section 4). However, this heating may be utilized to enhance decontamination potential and to reach pasteurization temperatures in selected cases potentially. Like, in a PL treatment at $59.4 \mathrm{~J} / \mathrm{cm}^{2}$, raspberries reached $75{ }^{\circ} \mathrm{C}$ from initial temperature of $30{ }^{\circ} \mathrm{C}$, which reduced Salmonella by 3.4 logs and E. coli O157:H7 by around three logs [28]. At higher fluence level $\left(72 \mathrm{~J} / \mathrm{cm}^{2}\right)$, the temperature rose to $80^{\circ} \mathrm{C}$. Due to the short duration of light pulses and relative short cumulative PL treatments (often limited by $12 \mathrm{~J} / \mathrm{cm}^{2}$ limitation), a steady-state thermal conduction is never achieved. Only a thin surface layer gets rapidly heated, sparing the inner layers of material, unlike the high overall temperature achieved in continuous systems [4]. Thus, PL treatment can be claimed to be a 'non-thermal' processing method because the inner layers are not prone to any temperature rise.

Overall, PL seems very effective for treating the surfaces of food products and packaging materials as its action on a thin surface layer is sufficient enough to destroy superficial vegetative cells [16]. PL is thus a rapid process of decontamination of food and packaging surfaces. PL processing offers several benefits over conventional processing like bulk of the product remains around the ambient temperatures due to shorter energy delivery time leading to no damage of inner parts of products. However, there have been reports on products getting heated due to prolonged exposure time, which needs to be mitigated. The PL systems are advantageous as flashlamps used to generate light pulses in PL systems do not require mercury, unlike continuous UV systems [20,21]. Apart from that, the PL systems also impart less risk from pathogens, and provide improved quality of the product than thermal processing, better economics while food processing due to low operational costs, greater flexibility, and leave no chemical/biochemical residues [23]. PL technology also stands out well as compared to other non-thermal technologies like HPP and PEF. In the case of HPP, food is needed to be usually packaged before treatment. Also, mild HPP treatment seems unable to inactivate spores, rather the process promotes germination [2]. On the other hand, PL effectively inactivates spores and decontaminate food and packaging materials. Food contact surface decontamination can also be achieved using PL [9,10]. PEF processing, although an excellent food preservation technique, has some limitations like lower processing capacities [7]. These challenges could be mitigated to a great extent using PL processing.

\section{Pulsed Light Equipment}

Initially, PL treatments were used for aesthetic applications like cosmetic, skin, and hair treatment areas. Later on, after approval of FDA in 1996, there has been tremendous development in the field of PL-based disinfection that has further been spread to food industries, pharmaceuticals, and water 
treatment plants. During the 1970s, Hiramoto [29] patented a pulsed UV light system for microbial inactivation [4]. Later on, studies were performed by Dunn et al. [30], who patented systems for sterilization of food and packaging materials. Since then, a major organization pioneering the PL based research in the world for food and drinking water decontamination was the Purepulse Technologies Inc., San Diego, California, a subsidiary of Xenon Corporation [23]. They acquired Hiramoto's patent and conducted further research to commercialize the PL equipment for food applications under the name PureBright ${ }^{\mathrm{TM}}$ system [4]. Subsequently, after several advancements in this field, the major companies manufacturing PL based decontamination systems are SteriBeam Systems from Germany, Wek-tec Systems from Germany, Claranor from France, Xenon Corp. from USA, and Solaris Disinfection Inc. from Canada. Table 2 summarizes the available PL systems for food decontamination.

A typical PL system works via the generation of light pulses using a pulse forming device. Pulses of light are produced by multiplying electrical power many folds by concentrating electrical energy in a capacitor over less than a second and releasing this energy in a relatively shorter duration (millionths or $10^{-6}$ of a second) to flashlamps. The flashlamps, filled with inert gases give off light in the form of flashes [12]. The pulses per second or pulse frequency varies mostly as $1-3 \mathrm{~Hz}$ [20]. The pulsed light systems basically have of three main units [13] as given in schematic diagram (Figure 5):

1. The power unit: Comprising electrical power supplier where high-voltage direct current (DC) power is obtained from low-voltage AC power.

2. Pulse configuration device: Comprising of high-voltage capacitors joined in parallel that concentrate energy from the power supplying unit in the charge cycle and release that during the discharge cycle, generating high electrical current. It is also connected to special high-power handling switches that perform on/off cycles of very short time converting the continuous low-electrical power into a pulsed high-electrical power.

3. The lamp unit: Comprising specially designed batteries of flashlamps containing gases that are excited due to pulsed high-electrical power from the pulsed configuration device. While transitioning to lower energy or ground states, they give off energy in the form of high intensity pulses. The high-intensity PL is delivered to the products by various lamp footprints and configurations.

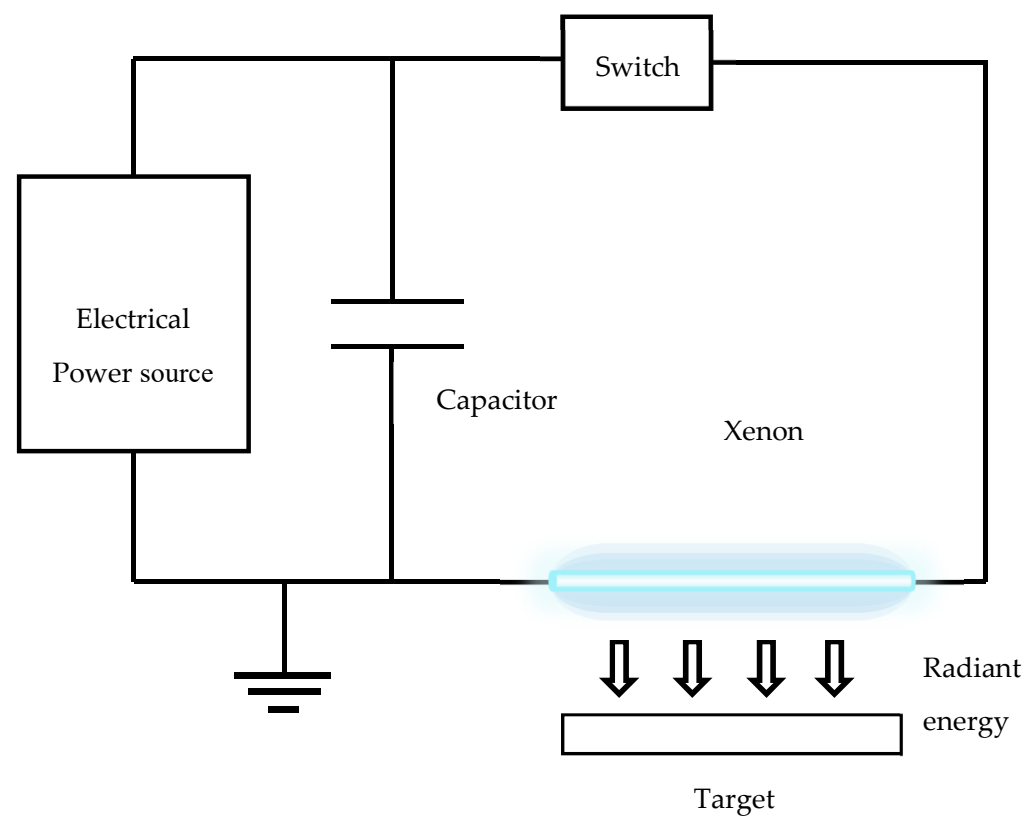

Figure 5. PL generation system. PL generation system consists of an electrical power source from where electric energy enters the capacitors, which accumulate the energy and get discharged into high-intensity light pulses. 
Table 2. PL systems for food decontamination.

\begin{tabular}{|c|c|c|}
\hline PL System & Characteristics & References \\
\hline \multirow{6}{*}{ Pure Bright ${ }^{\mathrm{TM}}$} & System for sterilizing liquid products (fruit juices) & \multirow{6}{*}{ [30] } \\
\hline & $\begin{array}{l}\text { Annular PL processing chamber with PL lamp inside and housed inside a highly reflective material } \\
\text { Tube-quartz made; arrangement-spherical, spiral, etc. } \\
\text { Electrodes- metallic (tungsten) electrodes } \\
\text { Flashlamps-filled with inert gases like xenon, krypton or a noble gases mixture at various pressures }\end{array}$ & \\
\hline & System for sterilization of a flexible film for aseptic packaging & \\
\hline & $\begin{array}{l}\text { Flexible film drawn through rollers into an absorption enhancement solution and then making them into a cylindrical tube } \\
\text { (with product filler and lamp inside) like structure, sealing the longitudinal and bottom seals. The lamp disinfects the } \\
\text { interior. Then product fills them and film is drawn forward. Then another seal is done transversally to totally seal the first } \\
\text { package and create bottom seal of next package. }\end{array}$ & \\
\hline & System for sterilizing preformed containers & \\
\hline & Packaging containers moved in line under the flashlamps and then pre-sterilized product fills them sequentially. & \\
\hline Wek-tec ${ }^{\circledR}$ & $\begin{array}{l}\text { The system had a stainless steel enclosure, } \\
\text { Test area dimensions: } 16 \mathrm{~cm} \text { wide } \times 12 \mathrm{~cm} \text { deep } \times 15 \mathrm{~cm} \text { high. } \\
\text { Flashlamps - linear; xenon filled } \\
\text { Fluence- } 0.1 \mathrm{~J} / \mathrm{cm}^{2} \text { to } 8 \mathrm{~J} / \mathrm{cm}^{2} \\
\text { Pulse duration - } 200 \mu \mathrm{s}\end{array}$ & [31] \\
\hline $\begin{array}{l}\text { Sterilization system for inner surfaces of } \\
\text { preformed containers }\end{array}$ & $\begin{array}{l}\text { The containers for packaging milk, juices placed on a conveyer belt, which move under the lamps that flash them. Then, the } \\
\text { container progresses forward. }\end{array}$ & [32] \\
\hline Bench top PL system & $\begin{array}{l}\text { System comprises a treatment chamber and a control module } \\
\text { Treatment chamber-stainless steel } \\
\text { Lamps-lamp house is at the top center and filled with Xenon gas. } \\
\text { The control module and light source connected by a control cable, to modulate the electric current to get specific pulse } \\
\text { repetition rate, pulse width and peak power. }\end{array}$ & [33] \\
\hline OneShot EN2/2143-1 unit & $\begin{array}{l}\text { 3-fluidized bed PUV system mounted with adjustable air nozzles to blow compressed air for fluidization of the food powders; } \\
\text { Water circulation in surrounding quartz jacket around the flash lamp to counter overheating and a reflecting cylinder. }\end{array}$ & [34] \\
\hline $\begin{array}{l}\text { RDT350 model, } \\
\text { La Calhene, USA }\end{array}$ & $\begin{array}{l}\text { Treatment chamber- } 250 \mathrm{~cm} \text { diameter } \\
\text { Lamps no.-8 xenon lamps arranged on the periphery of the chamber } \\
\text { Distance of samples from the xenon lamps was } 13.5 \mathrm{~cm} \\
\text { Lamps Fluence- } 1.5 \mathrm{~J} / \mathrm{cm}^{2} \\
\text { Pulse duration- } 300 \mu \mathrm{s} \\
\text { Wavelength-between } 200-1200 \mathrm{~nm}\end{array}$ & [13] \\
\hline
\end{tabular}


Table 2. Cont.

\begin{tabular}{|c|c|c|}
\hline PL System & Characteristics & References \\
\hline $\begin{array}{l}\text { Laboratory-scale PL system for } \\
\text { sterilization of infant foods }\end{array}$ & $\begin{array}{l}\text { Pulse discharge by quartz lamp with an oscilloscope to get the exponential decay pulse. } \\
\text { Water bath dissipates the heat generated during the operation }\end{array}$ & [35] \\
\hline $\begin{array}{l}\text { Intense PL system for sea foods } \\
\text { decontamination }\end{array}$ & $\begin{array}{l}\text { Lab scale system for decontamination of microorganisms in solid foods and sea foods. } \\
\text { Input voltage of } 220 \mathrm{~V} \text { alternating current (AC) supply rectified and transformed to a maximum voltage 50KV } \\
\text { Lamps- quartz lamp in which the Xenon gas is filled at } 450 \text { Torr pressure } \\
\text { Length- } 145 \mathrm{~mm} \\
\text { Outer diameter-7.14 mm } \\
\text { Wavelength- } 200-1100 \mathrm{~nm} \text { ranging from UV-C to IR }\end{array}$ & [36] \\
\hline Continuous-flow through PL system & For liquid food products like sugar syrup, juices & [37] \\
\hline SteriPulse ${ }^{\circledR}$ XL-3000 system & $\begin{array}{l}\text { Fluence: } 1.27 \mathrm{~J} / \mathrm{cm}^{2} \text { per pulse } \\
\text { Pulse duration-360 } \mu \mathrm{s} ; 3 \text { pulses/s } \\
\text { Broad spectrum UV with high germicidal power } \\
\text { Stainless steel sterilization chamber, removable lamp housing }\end{array}$ & [38] \\
\hline Claranor, France & PL system with multiple xenon lamps & [39] \\
\hline $\begin{array}{l}\text { The LytBot, Solaris Disinfection Inc., } \\
\text { Canada }\end{array}$ & PL system for Hospital Disinfection from & [40] \\
\hline
\end{tabular}




\section{Mechanism of Microbial Decontamination}

PL leads to the inactivation of microbes based on various extensive physicochemical processes. The microbial destruction is generally attributed to mechanisms like photo-chemical, photo-thermal, and photo-physical effects. These mechanisms are responsible for the inactivation of microorganisms independently or in combination with each other, depending on treatment conditions and target organisms [41]. Although photo-thermal and photo-physical effects might have an influence on inactivation, most works indicate the photo-chemical effect of light to be the main factor that drives the destruction of microorganisms.

Other than the physicochemical mechanisms, the spectral range and microbial morphology also govern the microbial destruction by PL to an extent. The effect of spectral range of light pulses has also been extensively studied. The spectral range found to be most efficient in microbial destruction was UV range [23], with UV-C component $(200-280 \mathrm{~nm}$ ) having the major role and a theoretical inactivation peak at $253.7 \mathrm{~nm}$ [41]. Wang et al. [42], however, reported that maximum reduction of E. coli took place at $270 \mathrm{~nm}$, in the UV-C range. Ramos-Villarroel et al. [43] also obtained greater inactivation in fresh-cut avocadoes by using the UV-C component rather than Vis-NIR spectra PL. However, the IR and visible regions of light radiation were not effective in PL-based inactivation process [23]. The IR and visible regions only had the antibacterial effect at higher powers [13].

Microbial morphology does not show consistency with respect to PL treatment. Rowan et al. [44] concluded the susceptibility of microorganisms to PL in the manner: Gram-negative bacteria > Gram-positive bacteria > fungal spores. In another study, Anderson et al. [45] observed similar trend in microbial sensitivity. Contrarily, Gómez-López et al. [46], however, did not report similar pattern of susceptibility in their studies. Spores have lower susceptibility than bacteria vegetative cells which was stated to be due to inherent DNA repair mechanisms of cell [45]. However, this tendency of repairing of DNA is highly affected by PL application as compared to continuous UV application, which is why PL is more effective [47].

Pigments in microorganisms can also be a determinant factor for PL efficacy. Anderson et al. [45] inferred that spore colors may have an effect on the action of PL. They showed that dark colored spores of the mold Aspergillus niger were resistant to PL rather than pink colored Fusrium culmorum, due to a greater absorption of UV-C light.

\subsection{Photo-Chemical Effect}

Researches firmly claim the chemical effect of PL to be the most important factor for microbial inactivation. UV absorption by DNA is claimed to be a major factor of germicidal effect of PL [41]. UV light shows antibacterial action by virtue of the formation of pyrimidine dimers, mostly by forming thymine dimers [1,41]. The UV light, particularly $253.7 \mathrm{~nm}$, corresponds to the threshold, which leads to dimer formation in microbial DNA. The light radiation of wavelengths less than or equal to $253.7 \mathrm{~nm}$ have the energy to carry out DNA dimerization [14]. These thymine dimers hinder the bacterial DNA replication process, thus resulting in the inactivation. Other than DNA dimerization, Gómez-López et al. [41] also reported that UV-C treatment of spores forms "spore photoproduct" like 5-thyminyl-5, 6-dihydrothymine and cyclo-butane pyrimidine dimers. UV light also gets absorbed by conjugated systems like carbon-carbon double bonds in DNA and protein molecules, thus causing structural alterations $[1,23]$. Wavelengths $>295 \mathrm{~nm}$ have been demonstrated to have capacity to destroy viruses. In another study, wavelengths $>400 \mathrm{~nm}$ were shown to destroy viruses due to the rupture of phage capsid [47].

Photosensitization is another facet of the photo-chemical effect. It involves the administration of certain photoactive compound (called 'photo-sensitizer') that gets accumulated in the target microorganism cells followed by light flashes [48]. The interaction of these compounds with light in excess of oxygen leads to the destruction of cells. Important advances have been made in photo-sensitized antimicrobial chemotherapy, like disinfection of the blood and blood products. Lukšiene et al. [49] carried out in vitro 5-aminolevulinic acid (ALA)-based photosensitization of 
Bacillus cereus and on the surface of packaging material. As a consequence of the photosensitization, several 'cytotoxic reactions' were reported to occur in the cells. Disruption of cellular membrane, enzymes inactivation and damage of DNA took place as a result [48]. Photosensitization-based microbial inactivation by PL could be a new area of application for light-based technologies.

\subsection{Photo-Thermal Effect}

The photo-thermal effects of light are shown by wavelengths corresponding to IR regions. Thermal effects of PL may add to inactivation of microorganisms if final temperature reaches pasteurization temperature. Although higher temperature by intense PL treatment might add to inactivation, it has been shown that the food sensory qualities are adversely affected. It has been previously emphasized in several studies that light pulses heat the surface layers of foods. The temperature of surface layer increases, which can destroy superficial pathogenic organisms. The heat can also penetrate inside of the food by conduction, but as mentioned, due to a short process the heat gets dissipated before it reaches the bulk. Nevertheless, a study by Hiramoto [29] showed that light pulses may be absorbed into A. niger cells when the molds heat instantaneously, which might lead to cell death. Dunn et al. [30] reported the possibility that light pulses to heat the food surfaces to destroy inhabiting bacteria. Changes in the structure and physiology of the cells might be the reason of cell death, what could be called photo-thermal stress. Wekhof et al. [31] showed that regional overheating of bacteria due to PL exposure may cause damage and rupture leading to an 'explosion' of cellular contents. They showed scanning electron-microscope (SEM) images of PL-treated A. niger spores, visualizing the release of overheated contents of spores. This happens due to the differential heating rate of cellular components leading to regional expansion and bursting of cell. Nicorescu et al. [50] demonstrated that in PL treatment of Bacillus subtilis, the cell wall got disrupted due to that photo-thermal stress. $\mathrm{Xu}$ and $\mathrm{Wu}$ [51] showed that, on PL treatment of E. coli, there were structural changes, possibly due to 'overheating, intercellular water vaporization and subsequent membrane disruption'. However, the studies showed the heat generation due to light pulses should only be a lesser contributing factor towards microbial inactivation, as compared to photochemical effects.

\subsection{Photo-Physical Effect}

The photo-physical effect of PL has also been identified to be a contributory factor for microbial inactivation. The tendency of high energy pulses to disrupt the cell structure was demonstrated by Krishnamurthy et al. [52]. They showed that Staphylococcus aureus cells suffered cell wall damage, shrinkage of plasma membrane, and leakage of cell organelles. In another study, Takeshita et al. [53] observed changes in cell shape and cell membrane, and the elusion of intracellular components and proteins in S. cerevisiae due to PL. Ramos-Villarroel et al. [54] also observed cytoplasmic damages to L. innocua and E. coli post PL treatment. Macias-Rodriguez et al. [55] carried out PL treatment of eggs to study photo-physical changes to E. coli cells and observed structural damage in the form of central depression in cells. These all studies show a contributory effect of photo-physical effect on microbial inactivation exclusive to PL. The structural damage tendency of PL is higher than that of continuous UV [47]. This might be due to application of high energy 'pulses' as compared to a continuous nature of the latter.

\section{Role of Pulsed Light Technology in Food Safety}

The PL technology has been shown to effectively inactivate several microorganisms like bacteria, yeasts, molds, and even viruses. The efficacy of its decontaminating several food substances has been well demonstrated and documented [56]. Several studies have been conducted in the past decades with respect to the application of PL in ensuring microbiological safety $[1,23,44,45,57]$. Some results from these and other in vitro studies and the decontamination of several solid and liquid food products, fresh fruits, and vegetables are shown in Tables 3 and 4, respectively. 
Table 3. The effects of PL treatments on microbial inactivation in vitro based on several published data.

\begin{tabular}{|c|c|c|c|c|c|}
\hline Microorganism & Media & $\begin{array}{l}\text { Pulse Energy } \\
\left(\mathrm{J} / \mathrm{cm}^{2}\right)\end{array}$ & $\begin{array}{l}\text { Treatment Time } \\
\qquad(\mu \mathrm{s})^{\mathrm{a}}\end{array}$ & $\log _{10}$ Reductions & Reference \\
\hline \multirow{2}{*}{ B. subtilis } & \multirow{2}{*}{ Tryptic soy broth } & 4 & 2 & 10 & \multirow{9}{*}{ [30] } \\
\hline & & 1 & 4 & 10 & \\
\hline \multirow{2}{*}{ E. coli } & \multirow{2}{*}{ Tryptic soy agar } & 4 & 1 & 10 & \\
\hline & & 1.5 & 2 & 10 & \\
\hline \multirow{2}{*}{ A. niger } & \multirow{2}{*}{ Potato dextrose agar } & 4 & 4 & 10 & \\
\hline & & 12 & 1 & 10 & \\
\hline \multirow{2}{*}{ S. aureus } & \multirow{2}{*}{ Tryptic soy agar } & 0.75 & 2 & 10 & \\
\hline & & 0.2 & 4 & 8 & \\
\hline Saccharomyces cerevisiae & Tryptic soy agar & 0.4 & 4 & 10 & \\
\hline E. coli $\mathrm{O} 157: \mathrm{H7}$ & \multirow{2}{*}{ Tryptone Soya-Yeast Extract Agar } & \multirow{2}{*}{3} & \multirow{2}{*}{$1-512$} & 6.82 & \multirow{2}{*}{ [21] } \\
\hline L. monocytogenes $4 \mathrm{~b}$ & & & & 6.25 & \\
\hline Pseudomonas aeruginosa & \multirow{7}{*}{ Tryptone Soya-yeast Extract Agar } & \multirow{7}{*}{3} & \multirow{7}{*}{20} & 5.8 & \multirow{7}{*}{ [44] } \\
\hline B. cereus & & & & 4.9 & \\
\hline E. coli O157:H7 & & & & 6.2 & \\
\hline L. monocytogenes & & & & 4.4 & \\
\hline S. enteritidis & & & & 5.6 & \\
\hline S. cerevisiae & & & & 4.9 & \\
\hline S. aureus & & & & 5.1 & \\
\hline $\begin{array}{l}\text { B. cereus, E. coli, } \\
\text { and } \\
\text { S. enteritidis }\end{array}$ & Tryptones soy-yeast extract broth & \multirow[t]{2}{*}{3} & \multirow[t]{2}{*}{85} & About 8 & \multirow[t]{2}{*}{ [45] } \\
\hline A. niger, Fusarium culmorum & Malt extract agar & & & 4.5 & \\
\hline A. niger & Buffer solution & 1 & 1000 & 4.8 & [31] \\
\hline \multirow{2}{*}{ Cryptosporidium parvum oocysts } & \multirow{2}{*}{$\begin{array}{l}\text { Solution containing approximately } 25 \times 10^{6} \\
\text { Cryptosporidium oocysts }\end{array}$} & 0.11 & - & \multirow{2}{*}{$3-5$} & \multirow{2}{*}{ [58] } \\
\hline & & 0.22 & - & & \\
\hline B. subtilis & Sterile deionized water & & $1-4$ & $2-5$ & [38] \\
\hline
\end{tabular}


Table 3. Cont

\begin{tabular}{|c|c|c|c|c|c|}
\hline Microorganism & Media & $\begin{array}{l}\text { Pulse Energy } \\
\left(\mathrm{J} / \mathrm{cm}^{2}\right)\end{array}$ & $\begin{array}{l}\text { Treatment Time } \\
(\mu \mathrm{s})^{\mathrm{a}}\end{array}$ & $\log _{10}$ Reductions & Reference \\
\hline S. cerevisiae & $\begin{array}{l}\text { Sterile potassium phosphate buffer } \\
\text { suspensions }\end{array}$ & 0.7 & 1200 & 6 & [53] \\
\hline Botrytis cinerea & Rose Bengal agar & - & $250 \mathrm{~s}$ & 3 & \multirow{2}{*}{ [59] } \\
\hline Monilinia fructigena & Malt extract agar & - & $250 \mathrm{~s}$ & 4 & \\
\hline \multirow{2}{*}{ S. aureus } & \multirow{2}{*}{ Baird-Parker agar plates } & 5.6 & 5400 & 7.5 & \multirow{2}{*}{ [25] } \\
\hline & & 5.6 & 5400 & 8.5 & \\
\hline Alicyclobacillus acidoterrestris & Orange serum agar & \multirow{19}{*}{7} & \multirow{19}{*}{1500} & $>5.2$ & \multirow{19}{*}{ [46] } \\
\hline B. circulans & Nutrient agar & & & $>4.1$ & \\
\hline Brochotrix thermosphacta & Nutrient agar & & & 3.1 & \\
\hline Leuconostoc mesenteroides & de Man, Rogosa and Sharpe agar & & & 4.0 & \\
\hline Photobacterium phosphoreum & Nutrient agar & & & $>4.4$ & \\
\hline P. fluorescens & Nutrient agar & & & 4.2 & \\
\hline Shewanella putrefaciens & Nutrient agar & & & 3.9 & \\
\hline Clostridium perfringens & Nutrient agar & & & $>2.9$ & \\
\hline B. cereus & Nutrient agar & & & 3.0 & \\
\hline E. coli O157:H7 & Nutrient agar & & & 4.7 & \\
\hline L. monocytogenes & Nutrient agar & & & 2.8 & \\
\hline S. typhimurium & Nutrient agar & & & 3.2 & \\
\hline Shigella flexnii & Nutrient agar & & & 3.8 & \\
\hline S. aureus & Nutrient agar & & & 5.1 & \\
\hline Yersina enterocolitica & Nutrient agar & & & 3.9 & \\
\hline A. flavus & Potato dextrose agar & & & 2.2 & \\
\hline Candida lambica & Yeast glucose choramphenicol & & & 2.8 & \\
\hline Rhodotorula mucilaginosa & Yeast glucose choramphenicol & & & $>2.8$ & \\
\hline B. cinerea & Potato dextrose agar & & & 1.2 & \\
\hline
\end{tabular}


Table 3. Cont

\begin{tabular}{|c|c|c|c|c|c|}
\hline Microorganism & Media & $\begin{array}{l}\text { Pulse Energy } \\
\quad\left(\mathrm{J} / \mathrm{cm}^{2}\right)\end{array}$ & $\begin{array}{l}\text { Treatment Time } \\
(\mu \mathrm{s})^{\mathrm{a}}\end{array}$ & $\log _{10}$ Reductions & Reference \\
\hline $\begin{array}{c}\text { Alternaria alternate, } \\
\text { A. niger, } \\
\text { B. cinerea, } \\
\text { F. oxysporum, } \\
\text { F. roseum, } \\
\text { M. fructicola, } \\
\text { Penicillium expansum, } \\
\text { P. digitatum, } \\
\text { Phytophthora citrophthora and } \\
\text { Rhizopus stolonifer }\end{array}$ & Solid culture media & Up to 0.2 & - & $\begin{array}{l}\text { Controlled } \\
\text { completely }\end{array}$ & [60] \\
\hline L. innocua & Clear liquid broth & 12 & - & $>6$ & [61] \\
\hline $\begin{array}{c}\text { S. aureus, E. coli NCTC 9001, } \\
\text { Methicillin-resistant S. aureus } \\
\text { (MRSA) LMG 15,975 and MRSA } \\
16 a\end{array}$ & Phosphate-buffered saline suspension & 630 & $30 \mathrm{~min}$ & $\begin{array}{l}5 \text { for S. aureus and } \\
\text { MRSA strains } \\
\text { Negligible for E. coli } \\
\text { NCTC } 9001\end{array}$ & [62] \\
\hline S. erterica serovar Enteritidis & Noble agar & 0.7 & - & 6.7 & [63] \\
\hline \multirow[b]{2}{*}{$\begin{array}{c}\text { E. coli ATCC } 25,922 \text { and E. coli } \\
\text { O157:H7 }\end{array}$} & Butterfield's phosphate buffer & \multirow[b]{2}{*}{13.1} & $4 \mathrm{~s}$ & $>8.5$ & \multirow[b]{2}{*}{ [64] } \\
\hline & Tryptic soy broth & & $\begin{array}{l}4 \text { s (12 pulses @ } 3 \\
\text { pulses/s) }\end{array}$ & 3.5 & \\
\hline $\begin{array}{c}\text { Murine norovirus (MNV-1) and } \\
\text { hepatitis A virus (HAV) }\end{array}$ & Viral suspensions & & $2 \mathrm{~s}$ & 5 & [65] \\
\hline L. innocua NCTC 11,288 & \multirow[b]{2}{*}{ Maximum recovery diluent } & \multirow[b]{2}{*}{28} & \multirow[b]{2}{*}{$8 \mathrm{~s}$} & 5.13 & \multirow[b]{2}{*}{ [66] } \\
\hline $\begin{array}{c}\text { E. coli } \\
\text { K12 DSM } 160\end{array}$ & & & & 4.67 & \\
\hline L. monocytogenes & Tryptone soy agar plate & 0.00175 & $180 \mathrm{~s}$ & 6 & [36] \\
\hline $\begin{array}{c}\text { B. subtilis } \\
\text { strain ATCC } 6633\end{array}$ & $\begin{array}{l}\text { Cell suspension } \\
\left(\mathrm{OD}_{580 \mathrm{~nm}}-0.8\right)\end{array}$ & 0.6 & - & 8.7 & [50] \\
\hline B. cereus and & \multirow{2}{*}{ Luria-Bertani Agar } & \multirow{2}{*}{1.95} & \multirow{2}{*}{$200 \mathrm{~s}$} & About 7 & \multirow{2}{*}[67]{} \\
\hline L. monocytogenes & & & & About 7 & \\
\hline
\end{tabular}


Table 3. Cont

\begin{tabular}{|c|c|c|c|c|c|}
\hline Microorganism & Media & $\begin{array}{l}\text { Pulse Energy } \\
\left(\mathrm{J} / \mathrm{cm}^{2}\right)\end{array}$ & $\begin{array}{l}\text { Treatment Time } \\
\qquad(\mu \mathrm{s})^{\mathrm{a}}\end{array}$ & $\log _{10}$ Reductions & Reference \\
\hline B. subtilis & $\begin{array}{l}\text { Nutrient agar for vegetative cells } \\
\text { Tryptic soy broth for spores } \\
\text { (cell density } 10^{9} \text { cells } / \mathrm{mL} \text { in both) }\end{array}$ & \multirow{2}{*}{12} & - & $\begin{array}{l}\text { About } 4 \text { of sporses } \\
>8 \text { for vegetative } \\
\text { cells }\end{array}$ & \multirow{2}{*}{ [68] } \\
\hline Geobacillus stearothermophillus & $\begin{array}{c}\begin{array}{c}\text { Nutrient agar for both vegetative cells and } \\
\text { spores }\end{array}\end{array}$ & & - & $\begin{array}{l}\text { About } 0.5 \text { for spores } \\
\text { About } 1 \text { for } \\
\text { vegetative cells }\end{array}$ & \\
\hline \multirow{2}{*}{ P. aeruginosa } & \multirow{2}{*}{ Plate count agar } & 1.13 & $30 \mathrm{~s}$ & 3.63 & \multirow{2}{*}{ [69] } \\
\hline & & About 11 & $300 \mathrm{~s}$ & About 6.5 & \\
\hline \multirow[b]{2}{*}{ L. monocytogenes Scott A } & Solid tryptic soy agar Petri plates & - & $20 s$ & 5 & \multirow[b]{2}{*}{ [1] } \\
\hline & $\begin{array}{l}0.1 \% \text { peptone water solution in whirl pak } \\
\text { bags }\end{array}$ & - & $93 \pm 5 \mathrm{~s}$ & 1 & \\
\hline Bacillus subtilis spores & Clear liquid medium & 0.017 & - & 1 & [70] \\
\hline Murine norovirus (MNV-1) and & \multirow{2}{*}{ Phosphate buffered saline } & \multirow{2}{*}{4.94} & - & $>5.8$ & \multirow{2}{*}{ [71] } \\
\hline Tulane virus (TV) & & & - & $>6.0$ & \\
\hline \multirow{2}{*}{ E. coli K-12 MG1655 } & \multirow{2}{*}{ Lysogeny broth (LB) } & $\begin{array}{c}76 \mathrm{~J} / \mathrm{cm}^{2} \\
\text { wavelength of } \\
190 \mathrm{~nm}\end{array}$ & - & 11 & \multirow{2}{*}{ [72] } \\
\hline & & $\begin{array}{c}95 \mathrm{~J} / \mathrm{cm}^{2} \\
\text { wavelength of } \\
240 \mathrm{~nm}\end{array}$ & & 6 & \\
\hline
\end{tabular}

a Time mentioned as $\mu$ s unless specified. 
Table 4. The effects of PL on some food products based on several published researches.

\begin{tabular}{|c|c|c|c|c|}
\hline Food Products & Pulse Energy $\left(\mathrm{J} / \mathrm{cm}^{2}\right)$ & Treatment Time $(\mu s) \mathrm{g}$ & $\log _{10}$ Reductions & Reference \\
\hline $\begin{array}{l}\text { Dry, non-creamed cottage } \\
\text { cheese curds }\end{array}$ & 16 & $\begin{array}{l}2 \text { pulses; } 0.5 \mu \mathrm{s} ; \text { Applied at } 3 \mathrm{~s} \\
\text { interval }\end{array}$ & $\mathrm{PVR}^{\text {a }}$ of $96.7 \%$ of Pseudomonas & \multirow{6}{*}{ [30] } \\
\hline \multirow[b]{2}{*}{ White bread rolls, hard crusted } & 16 & $0.5 \mu \mathrm{s}$ & PVR of $97.2 \%$ of mold spores & \\
\hline & 16 & $\begin{array}{l}2 \text { pulses; } 0.5 \mu \mathrm{s} ; \text { Applied at } 5 \mathrm{~s} \\
\text { interval }\end{array}$ & PVR of $99.8 \%$ of mold spores & \\
\hline $\begin{array}{l}\text { Cakes packaged in plastic } \\
\text { containers }\end{array}$ & 16 & $\begin{array}{l}3 \text { pulses; } 0.5 \mu \mathrm{s} ; \text { Applied at } 5 \mathrm{~s} \\
\text { interval }\end{array}$ & $\begin{array}{c}\text { No mold growth in treated samples during storage at room } \\
\text { temperature for } 10 \text { days, } \\
\text { Untreated samples-moldy in } 3 \text { days }\end{array}$ & \\
\hline $\begin{array}{l}\text { Whole fish, treated on the dark } \\
\text { side }\end{array}$ & 10 & 3 flashes; $0.5 \mu \mathrm{s}$ & $\begin{array}{l}1.93 \text { of coliforms } \\
2 \text { of psychrotrophs }\end{array}$ & \\
\hline Shrimp & $1-2$ & - & $\begin{array}{l}\text { Reduction by } 10-1000 \text { factors }^{\mathrm{b}} \text { of Listeria, Salmonella } \\
\text { Shelf-life increased by } 1 \text { week compared to untreated samples }\end{array}$ & \\
\hline White bread slices & Broad Spectrum PL & - & $\begin{array}{c}\text { Shelf life more than } 2 \text { weeks, } \\
\text { no surface molds }\end{array}$ & [73] \\
\hline Meat & Broad Spectrum PL & - & Reduction of Listeria and Salmonella & [73] \\
\hline Chicken wings & Broad Spectrum PL & - & 2 for Salmonella (inoculated) & [6] \\
\hline Frankfurters & 30 & - & 2 of L. innocua (inoculated) & [6] \\
\hline Retail meat & Broad Spectrum PL & - & $1-3$ of the lactic, total aerobic, enteric bacteria and Pseudomonas & [6] \\
\hline Commercial eggs & 4 & 8 & Up to 8 of $S$. Enteriditis (inoculated) & [7] \\
\hline Strawberries & 0.5 & $0.3 \mathrm{~ms}$ duration for pulses $4 \mathrm{~s}$ & No mold growth after 2 weeks storage & [74] \\
\hline Catfish fillets, pre-packaged & 0.25 & - & $\begin{array}{c}\text { Psychrotrophic and coliform bacteria not reduced just after treatment. } \\
\text { After storage of } 1 \text { week, psychrotrophic reduction to } 1 \text { log lesser than } \\
\text { untreated ones } \\
\text { Coliform reduction from about } 50 \text { to less than } 10 \mathrm{CFU} / \mathrm{g}\end{array}$ & [75] \\
\hline
\end{tabular}


Table 4. Cont.

\begin{tabular}{|c|c|c|c|c|}
\hline Food Products & Pulse Energy $\left(\mathrm{J} / \mathrm{cm}^{2}\right)$ & Treatment Time $(\mu \mathrm{s}) \mathrm{g}$ & $\log _{10}$ Reductions & Reference \\
\hline & 0.5 & - & $\begin{array}{l}\text { Psychrotrophic reduction to } 2 \text { log lower than untreated sample } \\
\text { Coliform reduction from about } 50 \text { to less than } 10 \mathrm{CFU} / \mathrm{g}\end{array}$ & \\
\hline Eggshells & 1.5 & - & $\begin{array}{l}3 \text { to } 6 \text { of Bacillus subtilis spores reduced with no. of pulses ranging } \\
\text { from } 2 \text { to } 6\end{array}$ & \multirow{3}{*}{ [76] } \\
\hline Cake & 1.5 & - & $\begin{array}{l}3 \text { to } 6 \text { of } A \text {. niger spores with } 2 \text { to } 6 \text { pulses; } \\
\text { Shelf-life up to } 6 \text { months for treated samples }\end{array}$ & \\
\hline Bread slices, Packed & 1.5 & - & Shelf life up to 5 months for treated samples & \\
\hline Milk & 25.1 & $114 \mathrm{~s}$ & $>2.0$ of Serratia marcescens & [77] \\
\hline Corn meal & $5.6^{\mathrm{c}}$ & $100 \mathrm{~s}$ & 4.93 of the spore of $A$. niger & [33] \\
\hline Strawberries & $7 \mathrm{~J}$ & 30 & No significant effect on growth of $M$. fructigena and B. cineria & [78] \\
\hline Alfalfa seeds & 5.6 & $90 \mathrm{~s}$ & 4.89 of E. coli $\mathrm{O} 157: \mathrm{H7}$ & [79] \\
\hline \multirow{2}{*}{ Clover honey } & 5.6 & $135 \mathrm{~s}$ & $2 \mathrm{~mm}$ deep samples: reduction of C. sporogenes spores to $73.9 \%$ & \multirow{2}{*}{ [22] } \\
\hline & 5.6 & $180 \mathrm{~s}$ & $8 \mathrm{~mm}$ deep samples: reduction of C. sporogenes spores to $89.4 \%$ & \\
\hline Wheat flour & 31.12 & - & $10.1 \%$ of $S$. cerevisiae & \multirow{2}{*}{ [35] } \\
\hline Black pepper & 31.12 & - & $44.5 \%$ of $S$. cerevisiae & \\
\hline Shredded spinach & \multirow{8}{*}{$7 \mathrm{~J}$} & \multirow{8}{*}{$180^{\mathrm{d}}$} & 0.9 of Mesophilic aerobic microorganisms & \multirow{8}{*}{ [80] } \\
\hline Grated celeriac & & & $0.21^{\mathrm{e}}$ of Mesophilic aerobic microorganisms & \\
\hline Chopped green paprika & & & 0.56 of Mesophilic aerobic microorganisms & \\
\hline Soybean sprouts & & & 0.65 of Mesophilic aerobic microorganisms & \\
\hline Shredded radicchio & & & 0.79 of Mesophilic aerobic microorganisms & \\
\hline Grated carrot & & & 1.64 of Mesophilic aerobic microorganisms & \\
\hline Shredded iceberg lettuce & & & $\begin{array}{l}2.04 \text { of Mesophilic aerobic microorganisms } \\
0.46 \text { of aerobic psychrotrophic counts }\end{array}$ & \\
\hline Shredded white cabbage & & & $\begin{array}{l}1.64 \text { of Mesophilic aerobic microorganisms } \\
0.54 \text { of aerobic psychrotrophic counts }\end{array}$ & \\
\hline \multirow{2}{*}{ Salmon fillets } & 5.6 & $60 \mathrm{~s}$ & 1.09 on skin side and 0.3 on muscle side and of E. coli O157:H7 & \multirow{2}{*}{ [81] } \\
\hline & 5.6 & $60 \mathrm{~s}$ & 1.02 on skin side and 0.74 on muscle side of L. monocytogenes Scott A & \\
\hline
\end{tabular}


Table 4. Cont.

\begin{tabular}{|c|c|c|c|c|}
\hline Food Products & Pulse Energy $\left(\mathrm{J} / \mathrm{cm}^{2}\right)$ & Treatment Time $(\mu \mathrm{s}) \mathrm{g}$ & $\log _{10}$ Reductions & Reference \\
\hline $\begin{array}{l}\text { Apples, kiwi, orange, lemon, } \\
\text { nectarine, peaches, pears, } \\
\text { raspberries, table grapes }\end{array}$ & Up to $0.500^{f}$ & $<10 \mathrm{~s}$ & $>5$ for fungi & [60] \\
\hline Milk & 1.27 & - & $0.55-7.26$ of S. aureus & [25] \\
\hline \multirow{2}{*}{ Blueberries } & 22.6 & $60 \mathrm{~s}$ & 4.3 of S. enterica & \multirow{2}{*}{ [82] } \\
\hline & 22.6 & $60 \mathrm{~s}$ & 2.9 of E. coli O157:H7 & \\
\hline \multirow{2}{*}{ Raspberries } & 59.4 & $60 \mathrm{~s}$ & 3.4 of Salmonella spp. & \multirow{4}{*}{ [28] } \\
\hline & 72.0 & $60 \mathrm{~s}$ & 3.9 of E. coli O157:H7 & \\
\hline \multirow{2}{*}{ Strawberries } & \multirow{2}{*}{64.8} & $60 \mathrm{~s}$ & 4.3 of Salmonella spp & \\
\hline & & $60 \mathrm{~s}$ & 3.3 of E. coli $\mathrm{O} 157: \mathrm{H7}$ & \\
\hline \multirow{2}{*}{ Chicken breast } & 5.4 & $200 \mathrm{~s}$ & 2.0 of $S$. Typhimurium & [83] \\
\hline & 16.8 & $15 \mathrm{~s}$ & 1.9 of $S$. Typhimurium & [84] \\
\hline Chicken frankfurters & 55.9 & $60 \mathrm{~s}$ & 1.6 of L. monocytogenes Scott A & [85] \\
\hline Vienna sausages & 9.4 & $4 \mathrm{~s}$ & 1.37 of L. innocua & [86] \\
\hline Apple juice & 8.8 & $3 \mathrm{~s}$ & 7.29 of E. coli ATCC 2592 & [64] \\
\hline \multirow{2}{*}{ Shell eggs } & 12 & - & 2.49 S. Enteriditis & [63] \\
\hline & 23.6 & $20 \mathrm{~s}$ & $>5.3$ of $S$. Enteriditis & [87] \\
\hline Infant milk powder & - & 9500 & 3 of L. monocytogenes & [35] \\
\hline Fresh-cut avocado & 0.4 & - & $\begin{array}{c}2.97 \text { of L. innocua } \\
3.33 \text { of E.Coli }\end{array}$ & [43] \\
\hline \multirow{2}{*}{ Apple juice } & \multirow{4}{*}{4} & \multirow[t]{2}{*}{-} & 4 of E.Coli & \multirow{4}{*}{ [88] } \\
\hline & & & 2.98 of L. innocua & \\
\hline \multirow{2}{*}{ Orange juice } & & \multirow[t]{2}{*}{-} & 2.90 of E. Coli & \\
\hline & & & 0.93 of L. innocua & \\
\hline Eggs (unwashed) & \multirow{2}{*}{$2.1-10.5$} & - & 4.9 of Salmonella & \multirow{2}{*}{ [89] } \\
\hline Eggs (washed) & & - & 5 of Salmonella & \\
\hline
\end{tabular}


Table 4. Cont

\begin{tabular}{|c|c|c|c|c|}
\hline Food Products & Pulse Energy $\left(\mathrm{J} / \mathrm{cm}^{2}\right)$ & Treatment Time $(\mu \mathrm{s}) \mathrm{g}$ & $\log _{10}$ Reductions & Reference \\
\hline Apple juice & \multirow{3}{*}{28} & \multirow{3}{*}{$8 \mathrm{~s}$} & $\begin{array}{l}>4.7 \text { of E. coli } \\
1.93 \text { of L. innocua }\end{array}$ & \multirow{3}{*}{ [66] } \\
\hline Orange juice & & & About 1 for both L. innocua and E. coli & \\
\hline Milk & & & $\begin{array}{l}1.06 \text { of E. coli } \\
0.51-0.84 \text { of L. innocua }\end{array}$ & \\
\hline Apple juice & 5.1 & 1.52 & 3.9 of E. coli & [90] \\
\hline Orange juice & 5.1 & $2.81 \mathrm{~s}$ & 2.42 of E. coli & [91] \\
\hline Apple juice & 5.1 & $300 \mathrm{~s}$ & 4.9 of E. coli & [92] \\
\hline $\begin{array}{c}\text { Plums, } \\
\text { tomatoes, cauliflowers, sweet } \\
\text { peppers }\end{array}$ & 5.4 & $0-200 \mathrm{~s}$ & $\begin{array}{l}\text { 1.1-1.5 of mesophiles } \\
1.3-1.8 \text { of B. cereus }\end{array}$ & [93] \\
\hline Fresh-cut watermelons & 12 & - & $\begin{array}{c}>3 \text { of E. coli } \\
2.79 \text { of L. innocua }\end{array}$ & [94] \\
\hline Fresh-cut mushrooms & 12 & - & $\begin{array}{c}3 \text { of E. coli } \\
2 \text { of L. innocua }\end{array}$ & [54] \\
\hline Milk (9.8\% total solids) & 8.4 & \multirow{5}{*}{-} & 2.5 of E. coli ATCC 25922 & \multirow{5}{*}{ [95] } \\
\hline $\begin{array}{l}\text { Concentrated milk ( } 45 \% \text { total } \\
\text { solids) }\end{array}$ & 8.4 & & $<1$ of E. coli ATCC 25922 & \\
\hline Skim milk & 14.9 & & 3.4 of E. coli ATCC 25922 & \\
\hline Milk (2\% fat) & 14.9 & & $>2.5$ of E. coli ATCC 25922 & \\
\hline Whole milk & 14.9 & & $>2.5$ of E. coli ATCC 25922 & \\
\hline $\begin{array}{l}\text { Ground spices (caraway, black } \\
\text { pepper and red pepper) }\end{array}$ & 10 & - & $\begin{array}{l}0.8 \text { in ground caraway and black pepper and } 1 \text { in ground red pepper } \\
\text { for B. subtilis }\end{array}$ & [50] \\
\hline Shrimp & \multirow{3}{*}{12.1} & \multirow{3}{*}{$1380 \mathrm{~s}$} & 2.4 of L. monocytogenes & \multirow{3}{*}{ [36] } \\
\hline Salmon & & & 2.1 of L. monocytogenes & \\
\hline Flatfish fillets & & & 1.9 of L. monocytogenes & \\
\hline $\begin{array}{l}\text { Ready-To-Eat dry cured meat } \\
\text { products }\end{array}$ & 11.9 & - & 1.5-1.8 of L. monocytogenes and S. enterica serovar Typhimurium & [96] \\
\hline
\end{tabular}


Table 4. Cont.

\begin{tabular}{|c|c|c|c|c|}
\hline Food Products & Pulse Energy $\left(\mathrm{J} / \mathrm{cm}^{2}\right)$ & Treatment Time $(\mu \mathrm{s}) \mathrm{g}$ & $\log _{10}$ Reductions & Reference \\
\hline \multirow{2}{*}{ Tomatoes } & 4 & \multirow{2}{*}{-} & 1 of surface microflora & \multirow{2}{*}{ [97] } \\
\hline & 2.2 & & 2.3 of S. cerevisiae & \\
\hline \multirow{4}{*}{ Strawberries } & \multirow{4}{*}{3.9} & \multirow{4}{*}{$200 \mathrm{~s}$} & 2.2 of Aerobic mesophilic bacteria & \multirow{4}{*}{ [67] } \\
\hline & & & 1 of yeasts and molds & \\
\hline & & & 1.5 of $B$. cereus & \\
\hline & & & 1.1 of L. monocytogenes & \\
\hline Orange Juice & \multirow{4}{*}{71.6} & \multirow{4}{*}{$60 \mathrm{~s}$} & $\begin{array}{l}\text { Up to } 1 \text { of } L \text {. innocua, } \\
\text { Up to } 2 \text { of E. coli } \\
\text { Up to } 1.5 \text { of } S . \text { Enteritidis } \\
\text { Up to } 0.5 \text { of } S . \text { cerevisiae }\end{array}$ & \multirow{4}{*}{ [98] } \\
\hline Strawberry juice & & & $\begin{array}{l}\text { Up to } 0.2 \text { of L. innocua, } \\
\text { Up to } 0.3 \text { of } S \text {. cerevisiae }\end{array}$ & \\
\hline Apple juice & & & $\begin{array}{l}\text { Up to } 4.5 \text { of L. innocua } \\
\text { Up to } 2 \text { of E. coli } \\
\text { Up to } 4 \text { of } S \text {. Enteritidis } \\
\text { Up to } 6 \text { of } S \text {. cerevisiae }\end{array}$ & \\
\hline Melon juice & & & $\begin{array}{l}\text { Up to } 6 \text { of L. innocua and E. coli } \\
\text { Up to } 5 \text { of S. Enteritidis } \\
\text { Up to } 7 \text { of S. cerevisiae }\end{array}$ & \\
\hline Blueberry wine & - & $60 \mathrm{~s}$ & $9.6 \pm 0.9$ of S. cerevisiae & \multirow{2}{*}{ [99] } \\
\hline White grape wine & - & $40 \mathrm{~s}$ & $5.9 \pm 0.5$ of $S$. cerevisiae & \\
\hline \multirow{2}{*}{ Blueberries } & \multirow{2}{*}{$5-56.1$} & \multirow{2}{*}{$360 \mu \mathrm{s}$} & 3.8- > 6.7 of E. coli $\mathrm{O} 157: \mathrm{H7}$ & \multirow{2}{*}{ [27] } \\
\hline & & & $4.8-5.7$ of Salmonella & \\
\hline Apple juice & 17.5 & - & 3 of L. brevis & [100] \\
\hline Raw milk & 26.25 & - & Up to 3.2 of total microbial count & [101] \\
\hline Packaged cheese & \multirow{2}{*}{53.4} & \multirow{2}{*}{$40 \mathrm{~s}$} & $\begin{array}{c}1.25 \text { of } P \text {. roqueforti } \\
2.98 \text { of Listeria monocytogenes }\end{array}$ & \multirow{2}{*}{ [102] } \\
\hline Unpackaged cheese & & & $\begin{array}{c}1.32 \text { of P. roqueforti } \\
3.08 \text { of L. monocytogenes }\end{array}$ & \\
\hline
\end{tabular}


Table 4. Cont.

\begin{tabular}{|c|c|c|c|c|}
\hline Food Products & Pulse Energy $\left(\mathrm{J} / \mathrm{cm}^{2}\right)$ & Treatment Time $(\mu \mathrm{s}) \mathrm{g}$ & $\log _{10}$ Reductions & Reference \\
\hline Fresh egg pasta & 1.75 & - & 3.3 of S. enterica & [103] \\
\hline Whey & 1.1 & - & 0.5 of L. innocua & [104] \\
\hline Pork and salmon & 30 & - & 0.96 (in pork) and 0.7 (in salmon) of aerobic microorganisms & [105] \\
\hline Cheese & $1.02-12.09$ & - & $\begin{array}{c}3.74 \pm 0.8 \text { of } P . \text { fluorescens } \\
5.41 \pm 0.1 \text { of } E . \text { coli ATCC } 25,922 \\
3.37 \pm 0.2 \text { of } L . \text { innocua }\end{array}$ & [106] \\
\hline Strawberries & 63.2 & \multirow{2}{*}{$60 \mathrm{~s}$} & $\begin{array}{c}2.4 \text { of E. coli O157:H7 } \\
2.2 \text { of Salmonella } \\
1.8 \text { of Murine norovirus (MNV-1) }\end{array}$ & \multirow{2}{*}{ [107] } \\
\hline Raspberries & 53.9 & & $\begin{array}{c}4.5 \text { of E. coli O157:H7 } \\
4.4 \text { of Salmonella } \\
3.6 \text { of Murine norovirus (MNV-1) }\end{array}$ & \\
\hline Endive salad and & \multirow{2}{*}{1} & - & $\begin{array}{c}2.34 \text { of } E \text {. coli } \\
2.54 \text { of } L . \text { innocua } \\
2.46 \text { of natural microflora }\end{array}$ & \multirow{2}{*}{ [108] } \\
\hline Mung bean sprouts & & - & $\begin{array}{c}1.91 \text { of E. coli } \\
1.55 \text { of L. innocua }\end{array}$ & \\
\hline Orange Juice & \multirow[b]{3}{*}{71.6} & \multirow[b]{3}{*}{$60 \mathrm{~s}$} & $\begin{array}{l}0.3-0.8 \text { of L. innocua, E. coli and S. Enteritidis } \\
\text { Upto } 0.5 \text { of S. cerevisiae }\end{array}$ & \multirow[b]{3}{*}{ [109] } \\
\hline Strawberry juice & & & 0.3-0.8 of L. innocua, E. coli and S. Enteritidis & \\
\hline Apple juice & & & $\begin{array}{c}1.6 \text { of } L . \text { innocua } \\
2.1 \text { of } \text { E. coli } \\
2.4 \text { of } S \text {. Enteritidis } \\
\text { Upto } 1 \text { of } S . \text { cerevisiae }\end{array}$ & \\
\hline Goat milk & 10 & - & 6 of E. coli & [110] \\
\hline \multirow{3}{*}{ Spinach } & 4 & & $0.5-2.2$ of initial microbial load & \multirow{3}{*}{ [111] } \\
\hline & 0.8 & - & $\begin{array}{l}1.85 \text { of L. innocua } \\
1.72 \text { of E. coli }\end{array}$ & \\
\hline & 12 & & $\begin{array}{l}2.6 \text { of L. innocua } \\
2.3 \text { of E. coli }\end{array}$ & \\
\hline
\end{tabular}


Table 4. Cont.

\begin{tabular}{|c|c|c|c|c|}
\hline Food Products & Pulse Energy $\left(\mathrm{J} / \mathrm{cm}^{2}\right)$ & Treatment Time $(\mu \mathrm{s}) \mathrm{g}$ & $\log _{10}$ Reductions & Reference \\
\hline \multirow{2}{*}{ Cantaloupe melon } & \multirow{2}{*}{15.6} & \multirow{2}{*}{-} & 1.39 of total plate count & \multirow{2}{*}[112]{} \\
\hline & & & 1.45 of yeasts and molds & \\
\hline \multirow{2}{*}{ Raspberries } & \multirow{2}{*}{28.2} & \multirow{2}{*}{$30 \mathrm{~s}$} & 4.5 of Salmonella & \multirow{2}{*}{ [51] } \\
\hline & & & 3.9 of E. coli O157:H7 & \\
\hline \multirow{2}{*}{ Powdered infant formula } & 0.199 & - & $>99 \%$ of L. innocua & \multirow{2}{*}{ [70] } \\
\hline & 0.398 & - & $>99 \%$ of $B$. subtilis spores & \\
\hline Coconut water & 19.2 & - & 5.2 of E. coli & [113] \\
\hline Strawberry & \multirow{2}{*}{22.5} & \multirow{2}{*}{$24 \mathrm{~s}$} & $\begin{array}{c}0.9 \pm 0.2 \text { of Murine norovirus } \\
1.9 \pm 0.3 \text { of } E . \text { coli O157:H7 } \\
2.1 \pm 0.4 \text { of Salmonella }\end{array}$ & \multirow{2}{*}[71]{} \\
\hline Blueberry & & & $\begin{array}{c}3.8 \pm 0.6 \text { of Murine norovirus } \\
5.7 \pm 1.2 \text { of } E . \text { coli O157:H7 } \\
4.2 \pm 0.2 \text { of Salmonella }\end{array}$ & \\
\hline $\begin{array}{c}\text { Tomatoes (slices packed in } \\
\text { sealed trays }\end{array}$ & 8 & $30 \mathrm{~min}$ & $\begin{array}{l}\text { About } 1.17 \text { of L. innocua CLIP11262 } \\
\text { About } 1.34 \text { of E. coli } 1.107\end{array}$ & [114] \\
\hline Fresh tomatoes & 8 & - & $\begin{array}{l}\text { Up to } 2 \text { of psychrophilic bacteria } \\
\text { Up to } 1.3 \text { of yeasts and molds }\end{array}$ & [115] \\
\hline Melons & 9 & - & The shelf life was extended 12 days & [116] \\
\hline Spinach & 15.75 & $15 \mathrm{~s}$ & 2.7 of E. coli $\mathrm{O} 157: \mathrm{H7}$ & [117] \\
\hline Strawberries & 3 & $10 \mathrm{~s}$ & Up to 0.8 for Salmonella & [118] \\
\hline Tomato stem scar & 31.5 & $30 \mathrm{~s}$ & 2.3 for S. enterica & [119] \\
\hline
\end{tabular}

a PVR = Percentage of viability reduction; calculated by the following formula: [\{1- (viable cell counts on treated sample/viable cell counts on control) $\times \times 100] ;{ }^{b}$ Factors: $10=1$ log reduction, $1000=3 \log$ reductions; ${ }^{\mathrm{c}}$ Maximum energy per pulse; ${ }^{\mathrm{d}}$ Treatment time in s/side; ${ }^{\mathrm{e}} 45 \mathrm{~s}$ treatment $/ \mathrm{side}^{\mathrm{f}}{ }^{\mathrm{f}}$ For Aspergillus niger fluence of $1.900 \mathrm{~J} / \mathrm{cm}^{2}{ }^{2} \mathrm{~g}$ Time mentioned as $\mu \mathrm{s}$ unless specified. 


\subsection{Microbial Decontamination in Food Products}

PL activity is different for solids and liquid food products. Solid foods absorb the PL and there is an exponential decay of light intensity as light goes on being absorbed as a function of the depth of food and its absorption coefficient. The extent to which PL penetrates the food products is termed its penetration depth, which has been defined as the distance where the light fluence rate is reduced to $37 \%(1 / \mathrm{e} ; \mathrm{e}=2.7128)$ of its initial value [14]. There are some other convention state penetration depths where light intensity is reduced to $10 \%$ of surface incident intensity. The penetration depth is defined as such because the reduction of light intensity to such a lower percentage is unable to carry out any inactivation beyond that depth. Heinrich et al. [120] reported that lower absorption coefficient, and higher absorption and transmission coefficients increase the penetration of PL. Uesugi and Moraru [86] demonstrated a penetration depth of $2.3 \mathrm{~mm}$ in Vienna sausages by the PL for a fluence of $9.4 \mathrm{~J} / \mathrm{cm}^{2}$ at $50.8 \mathrm{~mm}$ sample distance from lamp and achieving a $1.39 \mathrm{log}$ reduction in L. innocua. Vimont et al. [121] reported penetration of PL up to $10 \mathrm{~mm}$ depth in whey protein gels. Sauer and Moraru [64] obtained penetration depth of 41.7 and $15.9 \mathrm{~mm}$ for apple juice and cider respectively at fluences of 8.8 and $11.7 \mathrm{~J} / \mathrm{cm}^{2}$ and $50.8 \mathrm{~mm}$ sample distance from lamp. They also reported greater reductions of E. coli of more than 7 and 5.5 for apple juice and cider respectively. In another study, coconut water of depth $5 \mathrm{~mm}$ was successfully treated by PL at fluence level of $19.2 \mathrm{~J} / \mathrm{cm}^{2}$ and lamp distance of $50 \mathrm{~mm}$ [113]. Considering almost the same light intensity at the surface of any substrate, this indicates that PL has greater penetration in liquid foods than solid foods, with a steeper decay in light intensity in the solid foods.

Since in the case of solid foods products, the light does not penetrate well, PL application is mostly limited to surface inactivation and these are superficially decontaminated [38]. For, its superficial inactivation characteristics, PL would be effective for totally smooth products, where microorganisms would be totally exposed to light flashes. However, surfaces of solid foods are especially not smooth. Minute surface cracks and crevices could possibly shelter microorganisms, where light would not reach. Therefore, the entire superficial area of the foods has to be flashed so as to achieve the total decontamination of its surface despite surface unevenness, which makes the process very complex [16]. Several researches have been done on surface-treatment of fruits for microbial reductions. The possibility of PL to decontaminate the surfaces of fruits like strawberries and raspberries was demonstrated by Bialka and Demirci [28], with insignificant damage of fruits being reported. They treated raspberry surfaces using fluence of $72 \mathrm{~J} / \mathrm{cm}^{2}$ and obtained E. coli 0157:H7 and Salmonella reduction by 3.9 and 3.4 logs, respectively. Similarly, in strawberries, they obtained E. coli 0157:H7 and Salmonella reduction by 3.3 and $4.3 \mathrm{logs}$ respectively at $64.8 \mathrm{~J} / \mathrm{cm}^{2}$. However, very high fluence values were used. Marine and poultry products have also been treated efficiently by PL. Successful decontamination of surfaces was demonstrated for shrimp and fish [122]. Listeria on shrimp surface were reduced by 1-3 logs using 4-8 flashes of $1-2 \mathrm{~J} / \mathrm{cm}^{2}$ PL. Whereas fish treated with 3 PL flashes at intensity of $10 \mathrm{~J} / \mathrm{cm}^{2}$ gave reduction of about 2 logs of surface psychrotrophic bacteria. Paškevičiūte et al. [123] treated chicken surface with high-power PL of 1000 pulses and dose $5.4 \mathrm{~J} / \mathrm{cm}^{2}$ and reduced S. Typhimurium and L. monocytogenes by 2-2.4 logs and total aerobic mesophiles by 2 logs. Eggshells have also shown to be successfully decontaminated using light pulses. Exposure to PL of fluence levels of $2.1 \mathrm{~J} / \mathrm{cm}^{2}$ led to inactivation of Salmonella cells (5 log reductions per eggshell) on the egg surface [89]. Hierro et al. [63] performed PL treatment of shell eggs using $12 \mathrm{~J} / \mathrm{cm}^{2}$ fluence. They obtained about $2.49 \log$ reduction of $S$. Enteritidis on eggshell. The discrepancy between the results of the latter in spite of using higher fluence could be due to the employment of different cell recovery methods after treatment. Lasagabster et al. [89] used a shell rinse method for recovery of cells from shell surface, while Hierro et al. [63] used the shell crush method, indicating the former to be an inefficient cell recovery method [124]. Nevertheless, solid foods ranging from fruits, vegetables, and poultry to marine products have been treated successfully by PL. However, surface irregularities remain a challenge for their decontamination. 
Liquid sample treatment with PL is more challenging because microorganisms dwell in the whole volume of liquids. So, PL efficacy is particularly based on several factors like sample distance from lamp, exposure, turbidity, and optical and physicochemical properties of the sample, as well as sample depth $[16,110]$. A higher exposure time of liquid food leads to higher inactivation. Also, the sample distance from lamp determines treatment efficiency, i.e., lower the distance, the better. Optical properties like transparency, and turbidity affect the PL efficacy. Turbid liquid foods might hinder process efficiency because of light scattering. On the other hand, transparent liquids like clear juices are treated by PL efficiently [23]. Huffman et al. [125] treated water using PL at $0.25 \mathrm{~J} / \mathrm{cm}^{2}$ and obtained reductions in bacteria Klebsiella terrigena of more than 7.4 logs, and in viruses (Poliovirus and Rotavirus) and parasite (Cryptosporidium parvum) of above 4 logs. Milk was shown to be effectively treated by the exposure of $56 \mathrm{~s}$ to PL at a dose of $25.1 \mathrm{~J} / \mathrm{cm}^{2}$ [77]. S. aureus was reduced by $7.26 \mathrm{logs}$ at $1.27 \mathrm{~J} / \mathrm{cm}^{2}$ when subjecting milk to PL treatment [52]. This shows that the higher fluence values required for opaque food like milk as compared to water. Food composition also affects the PL processing. Miller et al. [95] carried out PL treatment of milk (concentrated milk, whole milk, skim milk) and showed that PL efficiency decreased as the content of milk fat and total solids increased. They obtained highest inactivation for skim milk and concluded that the presence of milk fat scattered light by fat globules. Other liquid foods like Apple juice and orange juice (inoculated with $E$ coli DH5- $\alpha$ and L. innocua 11,288) were subjected to continuous PL system with fluence of $4 \mathrm{~J} / \mathrm{cm}^{2}$ [88]. They the inactivation levels of 4.00 and $2.90 \mathrm{log}$ reductions respectively in apple and orange juices for Escherichia coli. Similarly, 2.98 and 0.93 logs for L. innocua were obtained for apple and orange juices, respectively. Higher turbidity and cloudiness in orange juice lowered the microbial inactivation by PL. Hillegas and Demirci [24] obtained $0.97 \log$ reductions of C. sporogenes spores inoculated in $2 \mathrm{~mm}$ depth of honey after 135 pulses intense PL treatment of $5.6 \mathrm{~J} / \mathrm{cm}^{2}$ per pulse. They concluded that microbial inactivation increased by varying the process parameters (lamp distance, number of pulses, sample depth), but complete inactivation was not achieved due to poor penetration of pulsed UV system in honey. Thus, the depth of liquid foods too affects the PL treatment. As light energy decreases with depth, thin film or profiles are suitable for PL processing. The physico-chemical properties like viscosity and density affect the flow properties of liquid foods and thus have to be considered while designing a continuous PL treatment system for them. Nevertheless, most of the studies show that PL is equally efficient and has great potential for the processing of several liquid foods. With the successful decontamination of clear liquids by PL, more research has to be extended towards turbid and opaque liquid foods.

\subsection{Food Contact Surface Decontamination}

Due to its superficial treatment characteristics, decontamination of food contact surfaces of equipment in processing plants could be achieved by PL technology [126]. However, there are only a few studies on the use of PL to sterilize equipment surfaces. Woodling and Moraru [127] investigated the role played by the surface topography of stainless steel on the efficacy of PL treatment. They obtained maximum reduction about 4 logs of L. innocua in stainless steel having varied surface finishes and concluded that PL efficacy was less in highly smooth as well as highly rough surfaces due to surface reflectivity and hiding of cells in the rough surfaces, respectively. Woodling and Moraru [9] also showed in another experiment the effect of spectral range on PL on different stainless-steel surfaces inoculated with L. innocua. While obtaining a maximum of about 4 logs reduction, they concluded that the UV component of light necessary for PL based microbial inactivation. In another study, Uesugi et al. [61] treated stainless steel coupons by PL of fluence $12 \mathrm{~J} / \mathrm{cm}^{2}$ to achieve about $4 \mathrm{log}$ reductions of L. innocua. Rajkovic et al. [10] showed that Pulsed UV light could be used for decontamination of the stainless-steel meat slicing knife (contacted with different meat products prior to treatment). However, meat composition played a role in the treatment efficiency. Complete inactivation of E. coli O157:H7 and L. monocytogenes by a $6.5 \mathrm{log}$ reduction per side of knife of was obtained in $60 \mathrm{~s}$ when the knife was used to cut meat having lower fat and protein content. In a different study, PL 
treatment was shown be effective to inactivate hepatitis A virus and murine norovirus (food-borne viruses) on food-handling surfaces [65]. Hepatitis A virus and Murine norovirus were inactivated up to $5 \operatorname{logs}$ in 3 and $2 \mathrm{~s}$, corresponding to fluences of 0.06 and $0.091 \mathrm{~J} / \mathrm{cm}^{2}$, respectively. In another study, PL treatment was employed for reduction of murine norovirus on both clean and fouled (by protein) stainless steel surface. The fluence level of $8.98 \mathrm{~J} / \mathrm{cm}^{2}$ for $6 \mathrm{~s}$ resulted in an approximately $4 \mathrm{log}$ reduction on a clean surface and a $2.6 \mathrm{log}$ reduction on a fouled surface [121]. This indicates that protein on surfaces interferes with viral inactivation by PL. However, higher fluence value and exposure time can suffice for fouled surfaces. PL also has a great potential for treatment of biofilms. Varying treatment conditions can help in obtaining greater efficiency in PL processing. Thus, the decontamination of food-contact and equipment surfaces is another promising area of PL application in the food industry.

\subsection{Food Package Decontamination}

There are several published results on the use of PL for the inactivation of microorganisms on food packaging materials. The characteristics of packaging material also determine PL treatment efficiency. The packaging materials should be transparent to UV light such as polyethylene, polypropylene, nylon, ethyl vinyl acetate (EVA), polybutylene, ethyl vinyl alcohol (EVOH) and Aclar for suitable PL treatment [4]. The application of PL has been shown to impart reductions of around 9 logs for vegetative microorganisms and above 7 logs for bacterial spores on the packaging materials surfaces [16]. In a study by Dunn et al. [122] of various packaging materials, surfaces were inoculated with $10-1000 \mathrm{CFU} / \mathrm{cm}^{2}$ and subjected to pulses of light. Single pulse of $1.25 \mathrm{~J} / \mathrm{cm}^{2}$ could inactivate S. aureus, while intensities more than $2 \mathrm{~J} / \mathrm{cm}^{2}$ were needed for inactivation of $B$. cereus and Aspergillus spp. spores. Molds species such as A. niger, A. cinnamomeus, A. repens, and C. herbarum, that are prevalent on the food packaging surface, after PL treatment, were reduced significantly by 2.7 logs on a paper-polyethylene packaging surface [128]. Currently, the form fill seal type or aseptic packaging films materials are sterilized in packaging machine by either $\mathrm{H}_{2} \mathrm{O}_{2}$ and/or UV light. An innovation suggested by the application of PL lies in the disinfection of such packaging materials in place of $\mathrm{H}_{2} \mathrm{O}_{2}$ or UV lamps [4].

\section{Effect on Food Quality Characteristics}

One of the main limitations of conventional processes like thermal processing is the impact on food quality aspects, including the taste, flavor, color, as well as nutritional and bioactive components. Being a non-thermal process, PL processing is expected to have no harmful impacts on the food quality while making it decontaminated. The potentiality of PL applications for food processing is thus affected by how it affects their color, taste, nutrients and other properties of food products. Various researches have been conducted to understand the effects of PL on food quality parameters. The effects of PL processing on various food quality attributes are discussed in following subsections.

\subsection{Effects on Organoleptic Properties (Color, Texture and Flavor) of Food Products}

Fruits and vegetables contain several phytochemicals and pigments which impart color and other sensory characteristics to them. It is very important to have a knowledge of the effects of PL on these product characteristics. In earlier studies conducted by Dunn et al. [30], PL was claimed to maintain sensory attributes without any significant changes. Many studies have emerged thereafter which have shown both similar and opposite results of effect of PL on the sensory properties of products like fruits and vegetables. Bialka and Demirci $[28,82]$ treated blueberries, raspberries and strawberries using pulsed UV light and observed insignificant color parameter and sensory changes, as well as no damages on the treated samples as compared to untreated ones. However, Bialka and Demirci [28] reported high inactivation at a high fluence level of $32.4 \mathrm{~J} / \mathrm{cm}^{2}$ at $3 \mathrm{~cm}$ sample distance from lamp but carried out color and sensory analyses at lower levels of 22.6 and $11.3 \mathrm{~J} / \mathrm{cm}^{2}$ at $8 \mathrm{~cm}$ distance. In another study, the color and firmness of strawberries remained acceptable after PL treatments [67]. Anugu [99] 
treated blueberry wine by PL and did not observe any significant effects on hue and lightness values of blueberry wine.

However, many studies have shown significant undesirable changes by PL treatment. Fine and Geravis [34] observed that undesirable color changes occurred on PL treatment of black pepper and wheat flour, which was apparent well before achieving the required microbial inactivation. Gómez-López et al. [80] reported discoloration in PL-treated iceberg lettuce and plastic like off-odor in shredded white cabbage immediately after treatment at 2700 pulses of $7 \mathrm{~J}$ intensity at $12.8 \mathrm{~cm}$ distance. This indicates a severe PL treatment might lead to significant changes to food quality. Slight color changes were also observed on PL treated apple juice [92]. Similarly, Ramos-Villarroel et al. [43,54,94] showed that the UV-C component of PL significantly hampered the firmness and color in fresh cut avocado, watermelons and mushrooms as UV-C wavelengths corresponding to higher energy have tendency to bring about undesirable sensory changes. There was a significant change in lightness value of the fresh-cut avocado and post-storage browning [43] after PL treatment with the main role of the UV-C region. There was a significant decrease in the color parameters and firmness of water-melons by PL application contributed significantly by UV-C. Color changes were attributed to decrease in carotenoid concentration [94] which might be due to the slight heating of samples and exposure to UV-C. A similar decrease in the lightness and firmness values of mushrooms was observed [54] after PL application. Abida et al. [15] also reported such effects of PL treatment on mushroom texture due to thermal damage by photo-thermal effect. Lukšiene et al. [93] affirmed that PL treatment affected the tactile properties of fruit and vegetables like texture and firmness, which was shown by Ramos-Villarroel et al. [43,54,94]. A slight decrease in the firmness of PL treated tomatoes was observed [115]. In PL treated mango pulp, there was $40 \%$ decrease in fruit firmness post-storage [129]. There was also significant improvement in color in PL treated mango peel and pulp after storage for seven days. Chroma values increased up to $140 \%$ in the peel and $130 \%$ in the pulp post-storage which was attributed to increments in their carotenoid concentration due to chlorophyll degradation, however, contradicting the study by Ramos-Villarroel et al. [94]. This might depend on the product matrix, e.g., in watermelon the PL decreases carotenoids, whereas in mango it leads to the conversion of chlorophyll into carotenoids. While severe treatment conditions are detrimental to product sensory quality, optimized treatment conditions at particular light intensity, number of pulses and lamp distance

Lipids in milk products might be quite susceptible to off-flavor generation auto-oxidation phenomenon, due to light. Slight aroma changes were observed in PL-treated goat milk [110] possibly due to photochemical changes in milk components and lipid oxidation. Krishnamurthy et al. [52] showed that adequately designed pulsed UV treatment process of milk does not induce lipid oxidation. Thus, properly designed fluid milk product PL treatment system are needed based on the optimized flow rate, fluence dosage, and residence time to minimize the effect on their color and sensory properties. Dunn [30] showed that no changes in color and taste occurred by PL in dry cottage cheese curd after severe treatment of $32 \mathrm{~J} / \mathrm{cm}^{2}$. Contrastingly, however, a smaller dose of $9.22 \mathrm{~J} / \mathrm{cm}^{2}$ adversely affected the sensory quality of cheese [130]. Color changes were significant for cheese treated by PL at $53.4 \mathrm{~J} / \mathrm{cm}^{2}$ for $40 \mathrm{~s}$ at $5 \mathrm{~cm}$ distance [102]. The color values $\mathrm{a}^{*}$ and $\mathrm{b}^{*}$ were significantly different from untreated ones, indicating changes in yellowness and redness.

The effects of PL application on sensory qualities of meat, sea-food, fish and other processed foods have been extensively studied. Dunn et al. [131] showed the sensory attributes of the PL-treated fish remained acceptable and close to control samples. They concluded that fish was acceptable even after 15 days of refrigerated storage. However, pulses of $2.5-5 \mathrm{~J} / \mathrm{cm}^{2}$ of UV light did not change color in packaged catfish fillets [75]. In another study, salmon fillets, on PL treatment for $30 \mathrm{~s} \mathrm{at} 3 \mathrm{~cm}$ distance and for $45 \mathrm{~s}$ at $5 \mathrm{~cm}$ distance showed excessive heating which resulted in color changes visually [81]. Paškevičiute and Lukšiene [83] did not observe any changes in color and flavor after PL treatment of $5.4 \mathrm{~J} / \mathrm{cm}^{2}$ in chicken breast meat. Keklik et al. [85] observed significant quality alterations in PL-treated chicken frankfurters. The fluence level up to $67 \mathrm{~J} / \mathrm{cm}^{2}$ for $60 \mathrm{~s}$ treatment induced darker, 
greenish and yellowish tint in the treated frankfurters. This could be due to application of higher fluence. The sensory qualities of PL-treated bologna slices were observed to be adversely affected [132]. Researchers noticed change in redness value and sensory attributes for dose above $2.1 \mathrm{~J} / \mathrm{cm}^{2}$. Wambura and Verghese [133] studied the impact of pulsed UV light on quality attributes of ham. They observed darkening of ham after processing and storage up to seven days with increasing $L^{*}$ value, and a slight increase in yellowness due to the increase in $b^{*}$ and decreasing redness (due to decrease in $\mathrm{a}^{*}$ ) value, possibly because of the accumulation of metmyoglobin on surface. They also observed a decrease in the firmness of meat post-processing. Pork and salmon, after PL treatment showed significant color and odor changes [105] post-PL treatment. Tomasevic and Rajkovic [134] found that PL treatment degraded the organoleptic properties of cooked meat, whereas it did affect the sensory attributes of dry cured meats and fermented sausages. The higher fluence dosage $\left(17 \mathrm{~J} / \mathrm{cm}^{2}\right)$ significantly changed the color parameter values of meat products. No significant differences in sensory and color quality were found for sea foods. Sulphur off-odor was detected in fresh egg pasta after PL treatment of $1.75 \mathrm{~J} / \mathrm{cm}^{2}$. However, no significant color change was observed [103]. As a matter of fact, fat content remains a decisive factor in PL treatment of meats with higher fat content leading to significant sensory changes. Fat however tends to increase the bacterial resistance to PL and thus fat rich meat products require treatment under severe conditions, jeopardizing their sensory characteristics.

\subsection{Effects on Physico-Chemical Properties of Foods}

The effects of PL processing on various physicochemical properties of food products like viscosity, $\mathrm{pH}$, functional properties have also been studied by several researchers. Shuwaish et al. [75] treated catfish fillets by PL of $2.5-5 \mathrm{~J} / \mathrm{cm}^{2}$ fluence and observed no effects on the shear forces. Marquenie et al. [78] did not observe any effect of PL on firmness of strawberries either singly (160 s treatment) or in combination with UV-C $\left(254 \mathrm{~nm}, 0.1 \mathrm{~J} / \mathrm{cm}^{2}\right.$ and $\left.120 \mathrm{~s} \mathrm{PL}\right)$. Krishnamurthy et al. [52] observed an increase in milk temperature up to $38{ }^{\circ} \mathrm{C}$ via the continuous flow PL treatment of milk, which led to fouling as well as alterations in milk quality. Kasahara et al. [110] noticed a slight decrease in viscosity, $\mathrm{pH}$ and density of milk after PL application. Palgan et al. [66] did not observe any significant change in ${ }^{\circ}$ Brix and $\mathrm{pH}$ of apple juice after PL treatment. Eggs treated with PL showed no drastic effect on the quality of egg albumin and on the sensory and functional properties [89]. The firmness and $\mathrm{pH}$ of the fresh-cut avocado after PL application however decreased; also, textural properties of fresh-cut watermelon and mushrooms $[43,54,94]$ were affected. PL also showed no impact on the soluble solids (in terms of $\left.{ }^{\circ} \mathrm{Brix}\right), \mathrm{pH}$, and non-enzymatic browning index. However, there was slight effect on the color of apple juice [92]. A slight decrease in $\mathrm{pH}$ and ${ }^{\circ}$ Brix and a rise in titratable acidity (in terms of $\mathrm{g}$ of anhydrous citric acid/100 g tomato) took place in tomatoes as a result storage of 20 days after PL treatment of $8 \mathrm{~J} / \mathrm{cm}^{2}$ [114]. Slight changes in cell wall integrity and weight decrease were observed in tomatoes after PL treatment of $8 \mathrm{~J} / \mathrm{cm}^{2}$ [115]. The physico-chemical properties are said to be affected in some studies and not in others. However, no conclusive results could be drawn, and it could be said that the effect of PL might be driven by product matrices.

\subsection{Effects on Nutrients and Bio-Active Components}

As already emphasized, PL processing tends to interact with the superficial layers of the food products which are in the vicinity of the light source. So, it does not appear to hamper their inner layers; hence, nutrients and bio-active components present within the inner layers remain intact as untreated samples. Researches have been done in plenty to study the effect on PL processing on food nutrients and bioactive components. On carrying out a nutritional evaluation of PL-treated frankfurters, Dunn et al. [6] observed no differences in proteins, vitamins like riboflavin and vitamin C, nitrosamine, or benzopyrene content compared to untreated samples, while a strong loss of riboflavin was reported in foods due to heat, light, and oxygen. Contrastingly, riboflavin content in products like chicken, beef and fish were did not decrease using pulsed UV treatment [135]. However, riboflavin and vitamin E contents were reduced only to $95 \%$ by four light pulses and to $85 \%$ by eight light pulses 
of their initial value, respectively. Wambura and Verghese [133] demonstrated pronounced oxidation in the treated ham samples in terms of the oxidative stability index (OSI) in hours, which showed a decreasing trend as storage period progressed. Abida et al. [15] reported that the PL treatment of mushroom reduced some phenolic compounds and vitamin $C$ content.

Lipid oxidation is induced in many food products post-PL processing and remains a challenge [102,105,123,133]. Paškevičiute et al. [123] observed PL induced lipid oxidation on chicken surfaces. The intensity of lipid oxidation in control and treated chicken samples showed a difference of $0.16 \mathrm{mg}$ malondialdehyde (MDA)/kg of chicken meat. Can et al. [102], in their PL treatment study of cheese at $5 \mathrm{~cm}$ distance for $40 \mathrm{~s}$, found that levels of lipid peroxidation as MDA concentration was found to be about 30.7 and $34.8 \mu \mathrm{g} \mathrm{MDA} / \mathrm{g}$ cheese in packaged condition and unpackaged conditions respectively, with unpackaged cheese undergoing higher oxidation due to exposure to oxygen. MDA levels in PL treated salmon and pork increased by $39.3 \%$ and $25.5 \%$, respectively after PL processing [105]. After PL treatment of milk, Elmnasser et al. [136] did not notice any lipid oxidation and changes in amino acid sequence and composition of proteins. Kasahara et al. [110] however talked about the possibility PL induced photochemical changes in milk lipids, proteins and oxidation of vitamins. Fernandez et al. [137] studied the protein oxidation of cheese as affected by PL, taking bovine serum albumin (BSA) as a model protein. They measured oxidation in terms of carbonyl content increase (nmol carbonyls/mg BSA), obtaining an increase in carbonyls above $10 \mathrm{nmol} / \mathrm{mg}$ BSA for fluence of $11.4 \mathrm{~J} / \mathrm{cm}^{2}$. Thus, lipid oxidation tends to remain a bottleneck in PL processing of fat rich products

Fruits like grapes and berries represent a rich source of phytonutrients and polyphenolic compounds. The retention of these compounds is necessary from the nutritional point of view. Impact of PL processing on these compounds has been a topic of continuous research. There was a decrease in total phenol and antioxidant activity (in Trolox equivalents) and impact on sensory attributes in $8 \mathrm{~s}$ (fluence of $1.17 \mathrm{~J} / \mathrm{cm}^{2}$ per pulse) PL treatment of apple juice [66]. However, light-based technologies, like continuous UV-C processing, have been shown to increase the concentration of phytochemicals in fruits. This could be due to the activation of plant defense response to severe treatments, which express the formation of the phytochemicals like phenolic compounds. In a study, level of resveratrol in grapes increased by more than 10-fold [138]. Given the similarities with UV, PL is claimed to have the similar effect of increasing phytochemicals [41]. Nevertheless, a study by Muñoz et al. [92] showed no effect on the antioxidant capacity at fluence level of $5.1 \mathrm{~J} / \mathrm{cm}^{2}$ and assisted thermosonication for orange juice. Pulsed UV exposure of blueberries enhanced their antioxidant capacity, phytochemicals and enzyme activity [99]. Pulsed UV treatment of blueberry wine enhanced the total anthocyanins (as mg of Cyanidin-3-glucoside equivalents (C-3-GE)/L of wine) and total flavonoids (as mg of Catechin equivalents (CE)/L of wine), but did not significantly reduce total phenolics (as mg of Gaelic acid equivalents (GAE)/L of wine) and antioxidant activity (as mmol Trolox equivalent/L of wine) [99]. There was increase in bio-active components like $\beta$-carotene, $\alpha$-carotene, and total lycopene contents with some lycopene isomerization in PL-treated tomatoes [97]. Green tomatoes showed increase in phenolic compounds, antioxidant activity, lycopene content, and total carotenoids after treatment [139]. Agüero et al. [110] observed a slight increase in polyphenolic content and antioxidant activity after the PL treatment of spinach. There was an increase in antioxidant activity in mango pulp [129]. The researchers found an increase in phenylalanine ammonia lyase enzyme content indicating increase in phenolic content. There was also little reduction in Vitamin C content in the mango pulp on storage after PL exposure. Braga et al. [140] shows that using pulsed UV as a pre-treatment for drying mangoes can reduce vitamins loss as compared to untreated dried mangoes. They found that the level of Vitamin C and carotenoids in untreated dried mangoes were $10 \%$ and $40 \%$ lower than mangoes treated with fluences between 3.6-10.8 J/ $\mathrm{cm}^{2}$. In addition, in samples subjected to fluences between 3.6 and $7.2 \mathrm{~J} / \mathrm{cm}^{2}$ the level of vitamins $B_{1}, B_{2}$ and $B_{5}$ risen by 10 to $25 \%$. However, Vitamin $\mathrm{B}_{6}$ was decreased by $40 \%-50 \%$ [140]. In a study, our research group showed the effect of PL on gallic acid solution (model solution of phenolic compounds in fruits and vegetables) [141]. It was 
observed that gallic acid solution turned brownish due to photo-degradation and a critical fluence of $3.82 \mathrm{~J} / \mathrm{cm}^{2}$ at $2 \mathrm{~cm}$ distance was identified, below which the gallic acid solution showed little to no degradation. Thus, as bioactive compounds being important from nutritional point of view, studies on the effect of PL on these compounds remain very important.

\subsection{Effects on Allergens, Toxins and Anti-nutritional Factors}

In several studies PL treatment is proposed to mitigate the allergenicity and toxicity of foods, which extends the applicability of PL treatment in food industry. For the first time, Chung et al. [142] showed the use of PL in mitigation of allergenicity of peanuts. They employed PL treatment for the prepare a peanut based product with hypoallergenic properties by reducing peanut allergens Ara $\mathrm{h} 1$ and Ara $\mathrm{h} 3$ to significant level. The major reason for changes in allergenicity of the proteins could be attributed to changes in structure, conformation and solubility of the protein subunits [143]. Pulsed UV treatment has ever since extended to other protein sources. PL treatment for $6 \mathrm{~min}$ led to a decrease in the major soy extracts allergen levels (i.e., glycinin, Gly $\mathrm{m} 6$ and $\beta$-conglycinin, Gly m5) probably through aggregation [144]. They also observed 50\% lowering in Immunoglobulin E (IgE) binding after treatment of $6 \mathrm{~min}$. In another study, Meinlschmidt et al. [143] showed reduction in immunoreactivity of Gly $\mathrm{m} 6$ and Gly $\mathrm{m} 5$ in soy protein isolate by $91 \%$ after $4 \mathrm{~min}$ treatment at $0.98 \mathrm{~J} / \mathrm{cm}^{2}$-s fluence. Also, PL treatment of $4 \mathrm{~min}$ was found to decrease the activity of the tropomyosin, a major allergen in shrimp, and to decrease the IgE-binding capacity of shrimp extract [145]. Li et al. [146] showed that PL exposure for 6-7 min reduced allergens of almond protein extracts and their IgE binding. Anugu [99] showed that PL was also effective in reducing antigenicity as well as the binding of IgE by isolated milk and egg proteins, in even shorter times [146]. Gluten immunoreactivity has been reduced by $50 \%$ using PL application at $26.26 \mathrm{~J} / \mathrm{cm}^{2}$ [147]. Oracjo et al. [148] treated $\beta$-lactoglobulin solution and reduced immunoreactivity of IgE and IgG by $45 \%$ and $85 \%$ after $16 \mathrm{~J} / \mathrm{cm}^{2}$ PL treatment.

Mycotoxins also have been inactivated by PL treatment in several studies. Mycotoxins like deoxynivalenol (DON), zearalenone, ochratoxin and aflatoxin (AF) B1 in solution were reduced by $72.5 \pm 1.1,84.5 \pm 1.9,98.1 \pm 0.2$ and $92.7 \pm 0.8 \%$, respectively [149]. In another study, Funes et al. [150] demonstrated that PL dose of up to $35.8 \mathrm{~J} / \mathrm{cm}^{2}$ reduced patulin level up to $22 \%$ in apple juice, whereas a $12 \mathrm{~J} / \mathrm{cm}^{2}$ treatment led to $51 \%$ lowering in patulin from apple purée. Furthermore, Wang et al. [151] showed that PL treatment completely eliminated the mutagenic activity of AF B1 and AF B2 in rice products like rough rice and rice bran. PL dose of $0.52 \mathrm{~J} / \mathrm{cm}^{2}$ per pulse for $80 \mathrm{~s}$ caused reduction by $75.0 \%$ and $39.2 \%$ of AF B1 and AF B2 in rough rice, respectively, while a $15 \mathrm{~s}$ treatment caused reduction in rice bran by $90.3 \%$ and $86.7 \%$ of $\mathrm{AF} B 1$ and $\mathrm{AF} B 2$, respectively. In a very recent study, concentration of DON in germinating barley was reduced by $35.5 \%$ using intense PL after 180 pulses for 1 min [152].

\subsection{Effects on Enzymes}

PL has also been studied for its effects on enzyme activity. The PL processing has a great potential to inactivate the food quality degrading enzymes. Broad spectrum PL may be useful to inactivate enzymes like polyphenol oxidase (PPO) of vegetables, which is related to enzymatic browning in these. Thus, PL treatment could be a solution to enzymatic browning in cut fruits like apples. Studies by Dunn et al. [30] showed that by exposing 2-5 flashes of full spectrum PL treatment of $3 \mathrm{~J} / \mathrm{cm}^{2}$, potato slice browning could possibly be inhibited. On the contrary, Abida et al. [15] reported PL treatment of mushroom induced some PPO activity leading to enzymatic browning due. Dunn et al. [30] showed that PL could also inactivate alkaline phosphatase found in various natural food products and milk. About $94 \%$ reduction in enzymatic activity of alkaline phosphatase was seen in PL treated milk [101]. In relation to pectin degrading enzymes, there was no significant effect on enzymes like pectin methyl esterase (PME) and polygalactouronase (PG) by PL treatment of tomatoes at $8 \mathrm{~J} / \mathrm{cm}^{2}$ [115]. Moreover, 20 days storage of both treated and untreated samples showed higher enzyme activity in the former and there was an approximately $40 \%$ reduction in the both enzymes' activity after 20 days. Contrastingly, a study has been done by Pellicer et al. [153] shows that PG can be degraded by $90 \%$ 
after applying $128 \mathrm{~J} / \mathrm{cm}^{2}$ on enzyme solution samples $(0.5 \mathrm{mg} / \mathrm{mL}, 20 \mathrm{~mL})$ through the photochemical process. Also, lipase enzyme, which is responsible for hydrolysis of lipids, was reduced to $10 \%$ by intense PL treatment [154].

\subsection{Effects on Post-Harvest Physiology of Fresh Produces}

PL has been also shown to have impact on the physiological processes in the fresh produces. The results in this regard are varying with treated products, but overall show that there seems to be an increase in plant respiration rate and increase in $\mathrm{CO}_{2}$ with increase in $\mathrm{O}_{2}$ as a response to PL application. An increase of $80 \%$ in the respiration rate was noticed in shredded Iceberg lettuce [79]. There was an increase in $\mathrm{CO}_{2}$ and ethanol due to respiration, but a reduction in $\mathrm{O}_{2}$ and ethylene production in the fresh-cut avocado after PL application [43]. There was increase in respiration rate in PL treated watermelon [94] and mushrooms [54]. There was also increase in ethylene production and ethanol in the watermelons [94]. Kramer [108] observed slight increase in respiration rate in endive salad. Agüero et al. [109] demonstrated little increase in respiration in spinach. Valdivia-Nájar et al. [114] demonstrated increase in $\mathrm{CO}_{2}$ and decrease in $\mathrm{O}_{2}$ in PL treated tomatoes.

Overall, the majority of these investigations tend to show that PL treatment has significant undesirable effects on the color and sensory attributes, especially in fresh produces like lettuce and mushrooms, meat products and fishes. which might be due to severe treatment conditions. The effects of PL on physico-chemical properties seem to vary product wise. Nevertheless, with respect to nutritional quality of foods, PL maintains the level of phytochemicals at over the period of post-harvest storage with demonstrated enhancement of phenolic compounds, carotenoids and antioxidant activity. Adding to the applications, PL has been shown to effectively mitigate the allergenicity and toxicity of several food products and inactivates select food quality degrading enzymes. The deleterious effect on sensory quality of food can be minimized by proper optimization of processing parameters of PL treatment like sample thickness, sample distance from lamp, fluence level, exposure time etc. The researchers $[54,80]$ have also suggested the use of chemicals like anti-browning agents or textural stabilizers for countering the observed decrease in firmness and browning of the food products, thereby enhancing their shelf life and maintaining the quality.

\section{Pulsed Light Technology for Enhancement of Bioactive Components in Foods}

PL treatment has been used in recent studies to show enhancement of bioactive compounds like phenolic compounds, carotenoids and antioxidants $[111,115,129,139]$. These are produced as a defense mechanism to external severities. However, light-based technologies like UV have been used for the enhancement of nutrients like vitamin D in mushrooms [155]. However, due to longer exposure time of 5-20 $\mathrm{min}$, there are discolorations in UV treated mushrooms [156]. PL processing being a shorter treatment has been shown to achieve the same objective with little to no discoloration. In an earlier study by Kalaras and Beelman [157], vitamin $\mathrm{D}_{2}$ was enhanced up to $824 \%$ daily value (DV-400 $\mathrm{IU} / 84 \mathrm{~g}$ being 100\% DV) per $84 \mathrm{~g}$ brown and white button mushrooms by application of $3.5 \mathrm{~J} / \mathrm{cm}^{2}$. Kalaras et al. [158] in a separate study, also observed Vitamin $\mathrm{D}_{2}$ content enhancement in white button mushrooms (Agaricus bisporus) was up to $27 \mu \mathrm{g} / \mathrm{g}$ dry weight using 18 pulses of pulsed UV light of $0.791 \mathrm{~J} / \mathrm{cm}^{2}$ per pulse for $1 \mathrm{~s}$ treatment. In another study, Koyyalamudi et al. [156] treated the white button mushrooms (Agaricus bisporus) passed in conveyor belts (at $31.7 \mathrm{~cm}$ distance) and enhanced Vitamin $D_{2}$ by $22.5 \mu \mathrm{g} / \mathrm{g}$ dry weight using nine pulses at $1.15 \mathrm{~J} / \mathrm{cm}^{2}$ per pulse. No visible discoloration was observed by them. Chen et al. [159] treated Pleurotus mushrooms with nine pulses of $1.15 \mathrm{~J} / \mathrm{cm}^{2}$ per pulse and enhanced vitamin $D_{2}$ by up to $2.78 \mu \mathrm{g} / \mathrm{g}$ fresh weight. They also treated mushroom powder under severe conditions of 60 pulses of $1.15 \mathrm{~J} / \mathrm{cm}^{2}$ per pulse at $5 \mathrm{~cm}$ distance and observed a vitamin $\mathrm{D}_{2}$ increment of up to $65.4 \mu \mathrm{g} / \mathrm{g}$ dry weight. Overall, the vitamin $\mathrm{D}_{2}$ enhancement varies proportionally with energy delivered during PL treatment. The vitamin $\mathrm{D}_{2}$ enhancement in mushrooms by PL was also patented recently $[160,161]$. 


\section{Future Challenges, Trends and Scope}

The commercialization of PL is possible only when the system is economical and affordable. Optimizing the important processing conditions to gain the desired log reduction level for specific food applications without jeopardizing quality would enable easier scaling-up. Any future efforts made to develop the PL process needs to be optimized based on the preliminary recommendations of U.S. FDA, viz.

- The total cumulative treatment, in terms of total fluence shall not exceed $12 \mathrm{~J} / \mathrm{cm}^{2}$

- The duration of pulses is $<2 \mathrm{~ms}$

- Typical pulse frequencies used in range of 1 to 20 pulses per second

Various challenges present themselves currently. For an effective PL treatment for microbial inactivation, the reflection coefficient of product surface should be low. Food products to be treated with PL should have good optical properties [4]. Food composition also plays important role in affecting PL efficacy. Solid foods and packaging materials must ideally be clear, smooth, and without roughness, pores, and grooves which could 'shadow' the microorganisms from the light [4]. Even highly smooth surfaces may also reflect light, rendering PL ineffective. These problems are further aggravated due to the ill-effects of overheating, which has hindered the treatment of carrots [80], alfalfa seeds [79], and raw salmon fillets [81]. Optimizing the desired photo-thermal based decontamination effect without unnecessary overheating of the product is an important challenge that needs to be addressed. Due to the shadowing effect, a PL system must be suitably developed for inactivating biofilms and granular materials like cereals, grains, and spices [47]. In the case of liquid foods, suspended particles or concentrated substrates in products like juice and milk can shield microorganisms, fat can minimize PL effectiveness, and liquids of low transmittance (like milk) will attenuate or scatter light before light could harm the microorganisms [47]. Proteinaceous and fat-rich food matrixes are not easily treated [80]. Although solid food products and clear juices are efficiently treated, opaque or turbid food treatment systems have to be devised based on research. Optimization of the processing parameters and solutions to these potential problems dictates the future of PL technology.

The PL technology has shown potential in reducing allergen levels in peanut [142] and other food products. In future, PL can be further explored to counter allergies related with other foods. While PL can be effective for treatment of smooth surfaces like packaging materials, one scope of research is on the development of PL for solid foods with rough surfaces, and granular materials like grains, seeds, spices etc. More research is needed on the applicability of photosensitization-based reduction of microbial load in foods. The enhancement of phytochemicals in food products apart from mushrooms could be an interesting scope for future research.

Major efforts are needed to address this engineering challenge of equipment development for novel PL applications including conveyor systems for solid food treatment and continuous flow-through systems for liquid foods. Pollock et al. [1] suggested a possibility of "thin profile PL treatment" of liquid foods, which is needed to be further explored. PL equipment with good penetration, like using fluidized beds for treating granular foods and multi-directional lights for uniform surface exposure [60], seems feasible in the future. PL could be employed for packaging films sterilization in aseptic packaging systems, as an alternative to the use of $\mathrm{H}_{2} \mathrm{O}_{2}$, and/or UV lamps. PL systems can be combined with other methods of food preservation, such as HHP or PEF also. PL equipment with cooling systems could be a solution to product heating problems.

Multiphysics modeling and simulation tools are important for process validation. and Continuous UV processing has been extensively studied and simulated using computation fluid dynamics (CFD). PL technology somehow resembles the UV treatment. A better understanding of PL interactions with foods and the design of continuous PL systems could be achieved by the CFD modeling and simulation. 


\section{Conclusions}

Pulsed light (PL) technology is a green, novel non-thermal technology that has huge potential to be employed for decontaminating food- and food-contact surfaces as well as packaging materials. Ever since the application of light pulses for food preservation, several terms have emerged, like pulsed UV light, high intensity broad spectrum pulsed light, pulsed white light etc., which are all the same as PL. PL has a great future in employment in the food industry for enhancement of keeping quality of food products by inactivating microorganisms such as L. monocytogenes within a few seconds.

However, PL cannot be used to sterilize food products due to their non-uniform surfaces and opacity, except to reduce their microbial load. Nevertheless, PL is one such technology, which has the capacity to tackle the undesirable effects of conventional thermal processing. PL is an apt method of decontamination for the surface of foods, packaging materials, equipment, and clear liquids. However, a challenge lies in the processing of particulate foods like grains, spices, and products having highly uneven surface due to the 'shadowing' of microorganisms. PL also has higher investment costs and has shown the possible heating of products. Therefore, PL based systems with a proper optimized system for minimizing PL induced sensory changes with minor modifications to allow for the processing of particulate foods as well as installed cooling jackets to minimize the heating will open new avenues for the process. Future research should be directed towards understanding the inactivation mechanisms by the PL of different bacterial strains, states, and morphologies, like Gram-positive and Gram-negative bacteria and different growth phases. Also, further attempts at the commercialization of the process have to be made in the future. Lastly, the enhancement of nutritional attributes due to PL light presents the opportunity of using PL as a bio-fortification technique, which might be of immediate demand in the natural health and nutraceutical sectors.

Author Contributions: Conceptualization, A.W., A.S. and A.P.S.; Data Curation, R.M., X.M. and A.W.; Formal analysis, R.M. and X.M.; Funding acquisition, A.P.S.; Investigation, R.M., A.S. and A.P.S.; Methodology, R.M., A.W. and A.P.S.; Project administration, A.S.; Supervision, A.P.S.; Writing-Original draft, R.M. and X.M.; Writing-Review \& editing, A.W. and A.P.S. All authors have read and agreed to the published version of the manuscript.

Funding: This research was funded by Natural Sciences and Engineering Research Council of Canada (NSERC) through the Discovery Grant (RGPIN-2018-04735) and Collaborative Research and Development Grant (CRDPJ 522364-17) in collaboration with Solaris Disinfection Inc., Mississauga, ON, Canada.

Acknowledgments: Authors thank the Natural Sciences and Engineering Research Council of Canada (NSERC) for financial support.

Conflicts of Interest: The authors declare no conflict of interest. The funders had no role in the design of the study; in the collection, analyses, or interpretation of data; in the writing of the manuscript, or in the decision to publish the result.

\section{References}

1. Pollock, A.M.; Pratap Singh, A.; Ramaswamy, H.S.; Ngadi, M.O. Pulsed light destruction kinetics of L. monocytogenes. LWT Food Sci. Technol. 2017, 84, 114-121. [CrossRef]

2. Rahman, M.S. Food Preservation: Overview. In Handbook of Food Preservation, 2nd ed.; Rahman, M.S., Ed.; CRC Press: Boca Raton, FL, USA, 2007; pp. 3-17.

3. Pratap Singh, A.; Singh, A.; Ramaswamy, H.S. Heat transfer phenomena during thermal processing of liquid particulate mixtures-A review. Crit. Rev. Food Sci. Nutr. 2015, 57, 1350-1364. [CrossRef] [PubMed]

4. Palmieri, L.; Cacace, D. High Intensity Pulsed Light Technology. In Emerging Technologies for Food Processing; Sun, D.-W., Ed.; Academic Press: Cambridge, MA, USA; Elsevier Ltd.: Amsterdam, The Netherlands, 2005; pp. 279-306.

5. Krishnamurthy, K.; Tewari, J.C.; Irudayaraj, J.; Demirci, A. Microscopic and spectroscopic evaluation of inactivation of Staphylococcus aureus by pulsed UV light and infrared heating. Food Bioprocess Technol. 2010, 3, 93-104. [CrossRef]

6. Dunn, J.; Ott, T.; Clark, W. Pulsed-light treatment of food and packaging. Food Technol. 1995, 49, 95-98.

7. Dunn, J. Pulsed light and pulsed electric field for foods and eggs. Poultry Sci. 1996, 75, 1133-1136. [CrossRef] 
8. Buchovec, I.; Paskeviciute, E.; Luksiene, Z.B. Photosensitization-based inactivation of food pathogen Listeria monocytogenes in vitro and on the surface of packaging material. J. Photoch. Photobio. B Biol. 2010, 99, 9-14. [CrossRef]

9. Woodling, S.E.; Moraru, C.I. Effect of spectral range in surface inactivation of Listeria innocua using broad-spectrum pulsed light. J. Food Prot. 2007, 70, 909-916. [CrossRef]

10. Rajkovic, A.; Tomasevic, I.; Smigic, N.; Uyttendaele, M.; Radovanovic, R.; Devlieghere, F. Pulsed ultraviolet light as an intervention strategy against Listeria monocytogenes and Escherichia coli O157:H7 on the surface of a meat slicing knife. J. Food Eng. 2010, 100, 446-451. [CrossRef]

11. Food and Drug Administration. Code of Federal Regulations; 21CFR179.41; FDA: Silver Spring, MD, USA, 1996.

12. Barbosa-Canovas, G.V.; Schaffner, D.W.; Pierson, M.; Zhang, Q.H. Pulsed light technology. J. Food Sci. 2000, 65 (Suppl. S8), 82-85. [CrossRef]

13. Elmnasser, N.; Guillou, S.; Leroi, F.; Orange, N.; Bakhrouf, A.; Federighi, M. Pulsed-light system as a novel food decontamination technology: A review. Can. J. Microbiol. 2007, 53, 813-821. [CrossRef]

14. Koutchma, T.; Forney, L.J.; Moraru, C.I. Ultraviolet Light in Food Technology: Principles and Applications; CRC Press: Boca Raton, FL, USA; Taylor \& Francis group: Abingdon, UK, 2009.

15. Abida, J.; Rayees, B.; Masoodi, F.A. Pulsed light technology: A novel method for food preservation. Int. Food Res. J. 2014, 21, 839-848.

16. Ortega-Rivas, E. Pulsed light Technology. In Non-Thermal Food Engineering Operations; Ortega-Rivas, E., Ed.; Springer: New York, NY, USA, 2012; pp. 263-272.

17. Mandal, R.; Shi, Y.; Singh, A.; Yada, R.Y.; Pratap Singh, A. Food Safety and Preservation. In Encyclopedia of Gastroenterology, 2nd ed.; Academic Press, Elsevier Ltd.: Amsterdam, The Netherlands, 2020; pp. 467-479.

18. Bintsis, T.; Litopoulou-Tzanetaki, E.; Robinson, R.K. Existing and potential applications of ultraviolet light in the food industry-A critical review. J. Sci. Food Agric. 2000, 80, 637-645. [CrossRef]

19. Gómez-López, V.M.; Bolton, J.R. An approach to standardize methods for fluence determination in bench-scale pulsed light experiments. Food Bioprocess Technol. 2016, 9, 1040-1048. [CrossRef]

20. Gómez-López, V.M. Pulsed Light Technology. In Handbook of Food Safety Engineering; Sun, D.-W., Ed.; Blackwell Publishing Ltd.: Singapore, 2012; pp. 643-665.

21. MacGregor, S.J.; Rowan, N.J.; McIlvaney, L.; Anderson, J.G.; Fouracre, R.A.; Farish, O. Light inactivation of food related pathogenic bacteria using a pulsed power source. Lett. Appl. Microbiol. 1998, 27, 67-70. [CrossRef] [PubMed]

22. Hillegas, S.L.; Demirci, A. Inactivation of Clostridium sporogenes in Clover Honey by Pulsed UV-Light Treatment; V. Manuscript FP 03 009; American Society of Agricultural and Biological Engineers: St. Joseph, MI, USA, 2003.

23. Bhavya, M.L.; Hebbar, H.U. Pulsed light processing of foods for microbial safety. Food Qual. Saf. 2017, 1, 187-202.

24. Zou, X.Y.; Lin, Y.L.; Xu, B.; Cao, T.C.; Tang, Y.L.; Pan, Y.; Gao, Z.-C.; Gao, N.Y. Enhanced inactivation of E. coli by pulsed UV-LED irradiation during water disinfection. Sci. Total Environ. 2019, 650, 210-215. [CrossRef] [PubMed]

25. Krishnamurthy, K.; Demirci, A.; Irudayaraj, J. Inactivation of Staphylococcus aureus by pulsed UV-light sterilization. J. Food Prot. 2004, 67, 1027-1030. [CrossRef]

26. Holck, A.L.; Liland, K.H.; Drømtorp, S.M.; Carlehög, M.; McLeod, A. Comparison of UV-C and Pulsed UV Light Treatments for Reduction of Salmonella, Listeria monocytogenes, and Enterohemorrhagic Escherichia coli on Eggs. J. Food Prot. 2018, 81, 6-16. [CrossRef]

27. Huang, Y.; Chen, H. A novel water-assisted pulsed light processing for decontamination of blueberries. Food Microbiol. 2014, 40, 1-8. [CrossRef]

28. Bialka, K.L.; Demirci, A. Efficacy of Pulsed UV-Light for the Decontamination of Escherichia coli O157:H7 and Salmonella spp. on Raspberries and Strawberries. J. Food Sci. 2008, 73, 201-207. [CrossRef]

29. Hiramoto, T. Method of Sterilization. U.S. Patent 4,464,336, 7 August 1984.

30. Dunn, J.E.; Clark, R.W.; Asmus, J.F.; Pearlman, J.S.; Boyer, K.; Painchaud, F.; Hofmann, G.A. Methods for Preservation of Foodstuffs. U.S. Patent 4,871,559, 3 October 1989.

31. Wekhof, A.; Trompeter, F.J.; Franken, O. Pulsed UV disintegration (PUVD): A new sterilisation mechanism for packaging and broad medical-hospital applications. In Proceedings of the First International Conference on Ultraviolet Technologies, Washington, DC, USA, 14-16 June 2001. 
32. Wekhof, A. Sterilising Packaging and Preserving Foodstuffs with Pulsed Light; Newsletter of International UV Association; International UV Association: Washington, DC, USA, 2002; Volume 4.

33. Jun, S.; Irudayaraj, J.; Demirci, A.; Geiser, D. Pulsed UV-light treatment of corn meal for inactivation of Aspergillus niger spores. Int. J. Food Sci. Technol. 2003, 38, 883-888. [CrossRef]

34. Fine, F.; Gervais, P. Efficiency of pulsed UV light for microbial decontamination of food powders. J. Food Prot. 2004, 67, 787-792. [CrossRef] [PubMed]

35. Choi, M.S.; Cheigh, C.I.; Jeong, E.A.; Shin, J.K.; Chung, M.S. Nonthermal sterilization of Listeria monocytogenes in infant foods by intense pulsed-light treatment. J. Food Eng. 2010, 97, 504-509. [CrossRef]

36. Cheigh, C.I.; Hwang, H.J.; Chung, M.S. Intense pulsed light (IPL) and UV-C treatments for inactivating Listeria monocytogenes on solid medium and seafoods. Food Res. Int. 2013, 54, 745-752. [CrossRef]

37. Ferrario, M.; Guerrero, S. Effect of a continuous flow-through pulsed light system combined with ultrasound on microbial survivability, colour and sensory shelf life of apple juice. Innov. Food Sci. Emerg. Technol. 2016, 34, 214-224. [CrossRef]

38. Sonenshein, A.L. Killing of Bacillus Spores by high-Intensity Ultraviolet Light; Xenon Corporation: Wilmington, MA, USA, 2003.

39. Rajkovic, A.; Tomasevic, I.; De Meulenaer, B.; Devlieghere, F. The effect of pulsed UV light on Escherichia coli O157: H7, Listeria monocytogenes, Salmonella typhimurium, Staphylococcus aureus and staphylococcal enterotoxin A on sliced fermented salami and its chemical quality. Food Control 2017, 73, 829-837. [CrossRef]

40. Lytbot. Meet the Lytbot-A highly Innovative First Line of Defence and Attack. Available online: https://solarislyt.com/the-lytbot/ (accessed on 18 March 2020).

41. Gómez-López, V.M.; Ragaerta, P.; Debeverea, J.; Devliegherea, F. Pulsed light for food decontamination: A review. Trends Food Sci. Technol. 2007, 18, 464-473. [CrossRef]

42. Wang, T.; MacGregor, S.J.; Anderson, J.G.; Woolsey, G.A. Pulsed ultra-violet inactivation spectrum of Escherichia coli. Water Res. 2005, 39, 2921-2925. [CrossRef]

43. Ramos-Villarroel, A.Y.; Martín-Belloso, O.; Soliva-Fortuny, R. Bacterial inactivation and quality changes in fresh-cut avocado treated with intense light pulses. Eur. Food Res. Technol. 2011, 233, 395-402. [CrossRef]

44. Rowan, N.J.; MacGregor, S.J.; Anderson, J.G.; Fouracre, R.A.; Mcllvaney, L.; Farish, O. Pulsed-light inactivation of food-related microorganisms. Appl. Environ. Microbiol. 1999, 65, 1312-1315. [CrossRef]

45. Anderson, J.G.; Rowan, N.J.; MacGregor, S.J.; Fouracre, R.A.; Farish, O. Inactivation of food-borne enteropathogenic bacteria and spoilage fungi using pulsed-light. IEEE Trans. Plasma Sci. 2000, 28, 83-88. [CrossRef]

46. Gómez-López, V.M.; Devlieghere, F.; Bonduelle, V.; Debevere, J. Factors affecting the inactivation of microorganisms by intense light pulses. J. Appl. Microinghbiol. 2005, 99, 460-470. [CrossRef] [PubMed]

47. Gómez-López, V.M.; Koutchma, T.; Linden, K. Ultraviolet and pulsed light processing of fluid foods. In Novel Thermal and Non-Thermal Technologies for Fluid Foods; Cullen, P.J., Tiwari, B.K., Valdramidis, V.P., Eds.; Academic Press: London, UK, 2012; pp. 185-223.

48. Lukšiene, Ž. New approach to inactivate harmful and pathogenic microorganisms: Photosensitization. Food Technol. Biotech. 2005, 43, 411-418.

49. Lukšiene, Ž.; Buchovec, I.; Paškevičiūtè, E. Inactivation of food pathogen Bacillus cereus by photosensitization in vitro and on the surface of packaging material. J. Appl. Microbiol. 2009, 107, 2037-2046. [CrossRef] [PubMed]

50. Nicorescu, I.; Nguyen, B.; Moreau-Ferret, M.; Agoulon, A.; Chevalier, S.; Orange, N. Pulsed light inactivation of Bacillus subtilis vegetative cells in suspensions and spices. Food Control 2013, 31, 151-157. [CrossRef]

51. $\mathrm{Xu}, \mathrm{W}$.; $\mathrm{Wu}, \mathrm{C}$. The impact of pulsed light on decontamination, quality, and bacterial attachment of fresh raspberries. Food Microbiol. 2016, 57, 135-143. [CrossRef] [PubMed]

52. Krishnamurthy, K.; Demirci, A.; Irudayaraj, J.M. Inactivation of Staphylococcus aureus in milk using flow-through pulsed UV-light treatment system. J. Food Sci. 2007, 72, 233-239. [CrossRef] [PubMed]

53. Takeshita, K.; Shibato, J.; Sameshima, T.; Fukunaga, S.; Isobe, S.; Arihara, K.; Itoh, M. Damage of yeasts induced by pulsed light irradiation. Int. J. Food Microbiol. 2003, 85, 151-158. [CrossRef]

54. Ramos-Villarroel, A.Y.; Aron-Maftei, N.; Martín-Belloso, O.; Soliva-Fortuny, R. The role of pulsed light spectral distribution in the inactivation of Escherichia coli and Listeria innocua on fresh-cut mushrooms. Food Control 2012, 24, 206-213. [CrossRef]

55. Macias-Rodriguez, B.; Yang, W.; Schneider, K.; Rock, C. Pulsed UV light as a postprocessing intervention for decontamination of hard-cooked peeled eggs. Int. J. Food Sci. Technol. 2014, 49, 2472-2480. [CrossRef] 
56. Oms-Oliu, G.; Martín-Belloso, O.; Soliva-Fortuny, R. Pulsed Light Treatments for Food Preservation. A Review. Food Bioprocess Technol. 2010, 3, 13-23. [CrossRef]

57. Roberts, P.; Hope, A. Virus inactivation by high intensity broad spectrum pulsed light. J. Virol. Methods 2003, 110, 61-65. [CrossRef]

58. Dunn, J.E.; Clark, R.W.; Bushnell, A.H.; Salisbury, K.J. Deactivation of Organisms Using High-Intensity Pulsed Polychromatic Light. U.S. Patent 6228332, 8 May 2001.

59. Marquenie, D.; Geeraerd, A.H.; Lammertyn, J.; Soontjens, C.; Van Impe, J.F.; Michiels, C.W.; Nicolaï, B.N. Combinations of pulsed white light and UV-C or mild heat treatment to inactivate conidia of Botrytis cinerea and Monilia fructigena. Int. J. Food Microbiol. 2003, 85, 185-196. [CrossRef]

60. Lagunas-Solar, M.C.; Piña, C.; MacDonald, J.D.; Bolkan, L. Development of pulsed UV light processes for surface fungal disinfection of fresh fruits. J. Food Prot. 2006, 69, 376-384. [CrossRef]

61. Uesugi, A.R.; Woodling, S.E.; Moraru, C.I. Inactivation kinetics and factors of variability in the pulsed light treatment of Listeria innocua cells. J. Food Prot. 2007, 70, 2518-2525. [CrossRef]

62. Maclean, M.; MacGregor, S.J.; Anderson, J.G.; Woolsey, G. High-intensity narrow-spectrum light inactivation and wavelength sensitivity of Staphylococcus aureus. FEMS Microbiol. Lett. 2008, 285, 227-232. [CrossRef]

63. Hierro, E.; Manzano, S.; Ordóñez, J.A.; de la Hoz, L.; Fernández, M. Inactivation of Salmonella enterica serovar enteritidis on shell eggs by pulsed light technology. Int. J. Food Microbiol. 2009, 135, 125-130. [CrossRef]

64. Sauer, A.; Moraru, C.I. Inactivation of Escherichia coli ATCC 25922 and Escherichia coli O157:H7 in apple juice and apple cider, using pulsed light treatment. J. Food Prot. 2009, 72, 937-944. [CrossRef]

65. Jean, J.; Morales-Rayas, R.; Anoman, M.-N.; Lamhoujeb, S. Inactivation of hepatitis A virus and norovirus surrogate in suspension and on food-contact surfaces using pulsed UV light (pulsed light inactivation of food-borne viruses). Food Microbiol. 2011, 28, 568-572. [CrossRef]

66. Palgan, I.; Caminiti, I.M.; Muñoz, A.; Noci, F.; Whyte, P.; Morgan, D.J.; Cronin, D.A.; Lyng, J.G. Effectiveness of High Intensity Light Pulses (HILP) treatments for the control of Escherichia coli and Listeria innocua in apple juice, orange juice and milk. Food Microbiol. 2011, 28, 14-20. [CrossRef]

67. Lukšiene, Ž.; Buchovec, I.; Viskelis, P. Impact of high-power pulsed light on microbial contamination, health promoting components and shelf life of strawberries. Food Technol. Biotechol. 2013, 51, 284-292.

68. Artíguez, M.L.; de Marañón, I.M. Inactivation of spores and vegetative cells of Bacillus subtilis and Geobacillus stearothermophilus by pulsed light. Innov. Food Sci. Emerg. Technol. 2015, 28, 52-58. [CrossRef]

69. Yi, J.Y.; Bae, Y.-K.; Cheigh, C.-I.; Chung, M.-S. Microbial inactivation and effects of interrelated factors of intense pulsed light (IPL) treatment for Pseudomonas aeruginosa. LWT Food Sci. Technol. 2017, 77, 52-59. [CrossRef]

70. Arroyo, C.; Dorozko, A.; Gaston, E.; O'Sullivan, M.; Whyte, P.; Lyng, J.G. Light based technologies for microbial inactivation of liquids, bead surfaces and powdered infant formula. Food Microbiol. 2017, 67, 49-57. [CrossRef]

71. Huang, Y.; Ye, M.; Cao, X.; Chen, H. Pulsed light inactivation of murine norovirus, Tulane virus, Escherichia coli O157:H7 and Salmonella in suspension and on berry surfaces. Food Microbiol. 2017, 61, 1-4. [CrossRef]

72. Faghihzadeh, F.; Anaya, N.M.; Hadjeres, H.; Boving, T.B.; Oyanedel-Craver, V. Pulse UV light effect on microbial biomolecules and organic pollutants degradation in aqueous solutions. Chemosphere 2019, 216, 677-683. [CrossRef]

73. Rice, J. Sterilizing with light and electrical impulses: Technological alternative to hydrogen peroxide, heat and irradiation. Food Process. 1994, 7, 66.

74. Dunn, J.; Ott, T.M.; Clark, W. Prolongation of Shelf-Life in Perishable Food Products. U.S. Patent 5489442 A, 6 February 1996.

75. Shuwaish, A.; Figueroa, J.E.; Silva, J.L. Pulsed-light-treated prepackaged catfish fillets. In Proceedings of the 2000 IFT Annual Meeting, Dallas, TX, USA, 10-14 June 2000.

76. Mimouni, A. Application de la lumiere pulsee en agroalimentaire. Ind. Aliment. Agricoles 2000, 8, 37-39.

77. Smith, W.L.; Lagunas-Solar, M.C.; Cullor, J.S. Use of pulsed ultraviolet laser light for the cold pasteurization of bovine milk. J. Food Prot. 2002, 65, 1480-1482. [CrossRef]

78. Marquenie, D.; Michiels, C.W.; Van Impe, J.F.; Schrevens, E.; Nicolaï, B.N. Pulsed white light in combination with UV-C and heat to reduce storage rot of strawberry. Postharvest Biol. Tec. 2003, 28, 455-461. [CrossRef]

79. Sharma, R.R.; Demirci, A. Inactivation of Escherichia coli O157:H7 on inoculated alfalfa seeds with pulsed ultraviolet light and response surface modeling. J. Food Sci. 2003, 68, 1448-1453. [CrossRef] 
80. Gómez-López, V.M.; Devlieghere, F.; Bonduelle, V.; Debevere, J. Intense light pulses decontamination of minimally processed vegetables and their shelf-life. Int. J. Food Microbiol. 2005, 103, 79-89. [CrossRef] [PubMed]

81. Ozer, N.P.; Demirci, A. Inactivation of Escherichia coli O157:H7 and Listeria monocytogenes inoculated on raw salmon fillets by pulsed UV-light treatment. Int. J. Food Sci. Technol. 2006, 41, 354-360. [CrossRef]

82. Bialka, K.L.; Demirci, A. Decontamination of Escherichia coli O157:H7 and Salmonella enterica on blueberries using ozone and pulsed UV-light. J. Food Sci. 2007, 72, 391-396. [CrossRef] [PubMed]

83. Paškevičiūte, E.; Lukšiene, Ž. High-power pulsed light for decontamination of chicken breast surface. Chemine Technologija 2009, 4, 53.

84. Keklik, N.M.; Demirci, A.; Puri, V.M. Decontamination of unpackaged and vacuum-packaged boneless chicken breast with pulsed UV-light. Poultry Sci. 2010, 89, 570-581. [CrossRef] [PubMed]

85. Keklik, N.M.; Demirci, A.; Puri, V.M. Inactivation of Listeria monocytogenes on unpackaged and vacuum-packaged chicken frankfurters using pulsed UV-light. J. Food Sci. 2009, 74, 431-439. [CrossRef]

86. Uesugi, A.R.; Moraru, C.I. Reduction of Listeria on Ready-to-Eat Sausages after Exposure to a Combination of Pulsed Light and Nisin. J. Food Prot. 2009, 72, 347-353. [CrossRef]

87. Keklik, N.M.; Demirci, A.; Patterson, P.H.; Puri, V.M. Pulsed UV light inactivation of Salmonella enteritidis on egg shells and its effects on egg quality. J. Food Prot. 2010, 73, 1408-1415. [CrossRef]

88. Pataro, G.; Muñoz, A.; Palgan, I.; Noci, F.; Ferrari, G.; Lyng, J.G. Bacterial inactivation in fruit juices using a continuous flow Pulsed Light (PL) system. Food Res. Int. 2011, 44, 1642-1648. [CrossRef]

89. Lasagabaster, A.; Arboleya, J.C.; Martínez de Marañón, I. Pulsed light technology for surface decontamination of eggs: Impact on Salmonella inactivation and egg quality. Innov. Food Sci. Emerg. Technol. 2011, 12, 124-128. [CrossRef]

90. Caminiti, I.M.; Palgan, I.; Noci, F.; Muñoz, A.; Whyte, P.; Cronin, D.A.; Morgan, D.J.; Lyng, J.G. The effect of pulsed electric fields (PEF) in combination with high intensity light pulses (HILP) on Escherichia coli inactivation and quality attributes in apple juice. Innov. Food Sci. Emerg. Technol. 2011, 12, 118-123. [CrossRef]

91. Muñoz, A.; Palgan, I.; Noci, F.; Morgan, D.J.; Cronin, D.A.; Whyte, P.; Lyng, J.G. Combinations of high intensity light pulses and thermosonication for the inactivation of Escherichia coli in orange juice. Food Microbiol. 2011, 28, 1200-1204. [CrossRef] [PubMed]

92. Muñoz, A.; Caminiti, I.M.; Palgan, I.; Pataro, G.; Noci, F.; Morgan, D.J.; Denis, A.; Cronin, D.A.; Whyte, P.; Ferrari, G.; et al. Effects on Escherichia coli inactivation and quality attributes in apple juice treated by combinations of pulsed light and thermosonication. Food Res. Int. 2012, 45, 299-305. [CrossRef]

93. Lukšiene, Ž.; Buchovec, I.; Kairyte, K.; Paškevičiūtė, E.; Viskelis, P. High-power pulsed light for microbial decontamination of some fruits and vegetables with different surfaces. J. Food Agric. Environ. 2012, 10, 162-167.

94. Ramos-Villarroel, A.Y.; Aron-Maftei, N.; Martín-Belloso, O.; Soliva-Fortuny, R. Influence of spectral distribution on bacterial inactivation and quality changes of fresh-cut watermelon treated with intense light pulses. Postharvest Biol. Technol. 2012, 69, 32-39. [CrossRef]

95. Miller, B.M.; Sauer, A.; Moraru, C.I. Inactivation of Escherichia coli in milk and concentrated milk using pulsed-light treatment. J. Dairy Sci. 2012, 95, 5597-5603. [CrossRef]

96. Ganan, M.; Hierro, E.; Hospital, X.F.; Barroso, E.; Fernández, M. Use of pulsed light to increase the safety of ready-to-eat cured meat products. Food Control 2013, 32, 512-517. [CrossRef]

97. Aguiló-Aguayo, I.; Charles, F.; Renard, C.M.; Page, D.; Carlin, F. Pulsed light effects on surface decontamination, physical qualities and nutritional composition of tomato fruit. Postharvest Biol. Technol. 2013, 86, 29-36. [CrossRef]

98. Ferrario, M.; Alzamora, S.M.; Guerrero, S. Inactivation kinetics of some microorganisms in apple, melon, orange and strawberry juices by high intensity light pulses. J. Food Eng. 2013, 118, 302-311. [CrossRef]

99. Anugu, A.K. Microbial Inactivation and Allergen Mitigation of Food Matrix by Pulsed Ultraviolet Light. Ph.D. Thesis, University of Florida, Gainesville, FL, USA, 2013. Available online: http://ufdc.ufl.edu/UFE0045406/ 00001 (accessed on 18 March 2020).

100. Ignat, A.; Manzocco, L.; Maifreni, M.; Bartolomeoli, I.; Nicoli, M.C. Surface decontamination of fresh-cut apple by pulsed light: Effects on structure, colour and sensory properties. Postharvest Biol. Technol. 2014, 91, 122-127. [CrossRef] 
101. Innocente, N.; Segat, A.; Manzocco, L.; Marino, M.; Maifreni, M.; Bortolomeoli, I.; Ignat, A.; Nicoli, M.C. Effect of pulsed light on total microbial count and alkaline phosphatase activity of raw milk. Int. Dairy J. 2014, 39, 108-112. [CrossRef]

102. Can, F.O.; Demirci, A.; Puri, V.M.; Gourama, H. Decontamination of hard cheeses by pulsed UV-light. J. Food Prot. 2014, 77, 1723-1731. [CrossRef] [PubMed]

103. Manzocco, L.; Maifreni, M.; Anese, M.; Munari, M.; Bartolomeoli, I.; Zanardi, S.; Suman, M.; Nicoli, M.C. Effect of pulsed light on safety and quality of fresh egg pasta. Food Bioprocess Tech. 2014, 7, 1973-1980. [CrossRef]

104. Artíguez, M.L.; de Marañón, I.M. Improved process for decontamination of whey by a continuous flow-through pulsed light system. Food Control 2015, 47, 599-605. [CrossRef]

105. Nicorescu, I.; Nguyen, B.; Chevalier, S.; Orange, N. Effects of pulsed light on the organoleptic properties and shelf-life extension of pork and salmon. Food Control 2014, 44, 138-145. [CrossRef]

106. Proulx, J.; Hsu, L.C.; Miller, B.M.; Sullivan, G.; Paradis, K.; Moraru, C.I. Pulsed-light inactivation of pathogenic and spoilage bacteria on cheese surface. J. Dairy Sci. 2015, 98, 5890-5898. [CrossRef]

107. Huang, Y.; Chen, H. Inactivation of Escherichia coli O157:H7, Salmonella and human norovirus surrogate on artificially contaminated strawberries and raspberries by water-assisted pulsed light treatment. Food Res. Int. 2015, 72, 1-7. [CrossRef]

108. Kramer, B.; Wunderlich, J.; Muranyi, P. Pulsed Light Decontamination of Endive Salad and Mung Bean Sprouts and Impact on Colour and Respiration Activity. J. Food Prot. 2015, 78, 340-348. [CrossRef]

109. Ferrario, M.; Alzamora, S.M.; Guerrero, S. Study of pulsed light inactivation and growth dynamics during storage of Escherichia coli ATCC 35218, Listeria innocua ATCC 33090, Salmonella Enteritidis MA44 and Saccharomyces cerevisiae KE162 and native flora in apple, orange and strawberry juices. Int. J. Food Sci. Technol. 2015, 50, 2498-2507. [CrossRef]

110. Kasahara, I.; Carrasco, V.; Aguilar, L. Inactivation of Escherichia coli in goat milk using pulsed ultraviolet light. J. Food Eng. 2015, 152, 43-49. [CrossRef]

111. Agüero, M.V.; Jagus, R.J.; Martín-Belloso, O.; Soliva-Fortuny, R. Surface decontamination of spinach by intense pulsed light treatments: Impact on quality attributes. Postharvest Biol. Technol. 2016, 121, 118-125. [CrossRef]

112. Koh, P.C.; Noranizan, M.A.; Karim, R.; Hanani, Z.A.N. Microbiological stability and quality of pulsed light treated cantaloupe (Cucumis melo L. reticulatus cv. Glamour) based on cut type and light fluence. J. Food Sci. Technol. 2016, 53, 1798-1810. [CrossRef] [PubMed]

113. Preetha, P.; Venugopal, A.P.; Varadharaju, N.; Kennedy, Z.J. Inactivation of Escherichia coli in Tender Coconut (Cocos nucifera L.) Water by Pulsed Light Treatment. Int. J. Curr. Microbiol. Appl. Sci. 2017, 6, 1453-1461. [CrossRef]

114. Valdivia-Nájar, C.G.; Martín-Belloso, O.; Giner-Seguí, J.; Soliva-Fortuny, R. Modeling the Inactivation of Listeria innocua and Escherichia coli in Fresh-Cut Tomato Treated with Pulsed Light. Food Bioprocess Tech. 2017, 10, 266-274. [CrossRef]

115. Valdivia-Nájar, C.G.; Martín-Belloso, O.; Soliva-Fortuny, R. Impact of pulsed light treatments and storage time on the texture quality of fresh-cut tomatoes. Innov. Food Sci. Emerg. Technol. 2018, 45, 29-35. [CrossRef]

116. De Sousa, A.E.D.; de Almeida Lopes, M.M.; Moreira, A.D.R.; Macedo, J.J.N.; Moura, C.F.H.; de Aragão, F.A.S.; Zocolo, G.J.; de Miranda, M.R.A.; de Oliveira Silva, E. Induction of postharvest resistance in melon using pulsed light as abiotic stressor. Sci. Hortic. 2019, 246, 921-927. [CrossRef]

117. Mukhopadhyay, S.; Sokorai, K.; Ukuku, D.O.; Fan, X.; Olanya, M.; Juneja, V. Effects of pulsed light and sanitizer wash combination on inactivation of Escherichia coli O157: H7, microbial loads and apparent quality of spinach leaves. Food Microbiol. 2019, 82, 127-134. [CrossRef]

118. Cao, X.; Huang, R.; Chen, H. Evaluation of Food Safety and Quality Parameters for Shelf Life Extension of Pulsed Light Treated Strawberries. J. Food Sci. 2019, 84, 1494-1500. [CrossRef]

119. Leng, J.; Mukhopadhyay, S.; Sokorai, K.; Ukuku, D.O.; Fan, X.; Olanya, M.; Juneja, V. Inactivation of Salmonella in cherry tomato stem scars and quality preservation by pulsed light treatment and antimicrobial wash. Food Control 2020, 110, 107005. [CrossRef]

120. Heinrich, V.; Zunabovic, M.; Varzakas, T.; Bergmair, J.; Kneifel, W. Pulsed Light Treatment of Different Food Types with a Special Focus on Meat: A Critical Review. Crit. Rev. Food Sci. Nutr. 2015, 56, 591-613. [CrossRef] [PubMed] 
121. Vimont, A.; Fliss, I.; Jean, J. Efficacy and mechanisms of murine norovirus inhibition by pulsed-light technology. Appl. Environ. Microbiol. 2015, 81, 2950-2957. [CrossRef]

122. Dunn, J.E.; Clark, R.W.; Asmus, J.F.; Pearlman, J.S.; Boyer, K.; Pairchaud, F.; Hofmann, G. Methods for Preservation of Foodstuffs. U.S. Patent 5,034,235, 23 July 1991.

123. Paškevičiūte, E.; Buchovec, I.; Lukšiene, Ž. High-power pulsed light for decontamination of chicken from food pathogens: A study on antimicrobial efficiency and organoleptic properties. J. Food Safety 2011, 31, 61-68. [CrossRef]

124. Musgrove, M.T.; Jones, D.R.; Northcutt, J.K.; Cox, N.A.; Harrison, M.A. Shell rinse and shell crush methods for the recovery of aerobic microorganisms and Enterobacteriaceae from shell eggs. J. Food Prot. 2005, 68, 2144-2148. [CrossRef] [PubMed]

125. Huffman, D.E.; Slifko, T.R.; Salisbury, K.; Rose, J.B. Inactivation of bacteria, virus and Cryptosporidium by a point-of-use device using pulsed broad spectrum white light. Water Res. 2000, 34, 2491-2498. [CrossRef]

126. Food Safety Magazine. Shedding Light on Food Safety: Applications of Pulsed Light Processing. 2014. Available online: https://www.foodsafetymagazine.com/magazine-archive1/junejuly-2014/shedding-lighton-food-safety-applications-of-pulsed-light-processing/ (accessed on 4 October 2019).

127. Woodling, S.E.; Moraru, C.I. Influence of Surface Topography on the Effectiveness of Pulsed Light Treatment for the Inactivation of Listeria innocua on Stainless-steel Surfaces. J. Food Sci. 2005, 70, 345-351. [CrossRef]

128. Turtoi, M.; Nicolau, A. Intense light pulse treatment as alternative method for mould spores destruction on paper-polyethylene packaging material, J. Food Eng. 2007, 83, 47253. [CrossRef]

129. Lopes, M.M.; Silva, E.O.; Canuto, K.M.; Silva, L.M.; Gallão, M.I.; Urban, L.; Fernando Ayala-Zavala, J.; Miranda, M.R.A. Low fluence pulsed light enhanced phytochemical content and antioxidant potential of 'Tommy Atkins' mango peel and pulp. Innov. Food Sci. Emerg. Technol. 2016, 33, 216-224. [CrossRef]

130. Proulx, J.; Sullivan, G.; Marostegan, L.F.; VanWees, S.; Hsu, L.C.; Moraru, C.I. Pulsed light and antimicrobial combination treatments for surface decontamination of cheese: Favorable and antagonistic effects. J. Dairy Sci. 2017, 100, 1664-1673. [CrossRef]

131. Dunn, J.; Clark, W.R.; Asmus, J.F.; Pearlman, J.S.; Boyer, K.; Pairchaud, F.; Hofmann, G. Methods and Apparatus for Preservation of Foodstuffs. U.S. Patent Application No. WO 88/03369, 3 October 1989.

132. Hierro, E.; Manzano, S.; Ordóñez, J.A.; de la Hoz, L.; Fernández, M. Efficacy of pulsed light for shelf-life extension and inactivation of Listeria monocytogenes on ready-to-eat cooked meat products. Innov. Food Sci. Emerg. Technol. 2011, 12, 275-281. [CrossRef]

133. Wambura, P.; Verghese, M. Effect of pulsed ultraviolet light on quality of sliced ham. LWT Food Sci. Technol. 2011, 44, 2173-2179. [CrossRef]

134. Tomasevic, I.; Rajkovic, A. The Sensory Quality of Meat, Game, Poultry, Seafood and Meat Products as Affected by Intense Light Pulses: A Systematic Review. Procedia Food Sci. 2015, 5, 285-288. [CrossRef]

135. Tonon, F.; Agoulon, A. Lumiere pulse, principe et application au cas des solutions liquides. In Industries Agro-Alimentaires, la Conservation de Demain, 4th ed.; Commission Européenne: Talence, France, 20 November 2003.

136. Elmnasser, N.; Dalgalarrondo, M.; Orange, N.; Bakhrouf, A.; Haertlé, T.; Federighi, M.; Chobert, J.M. Effect of pulsed-light treatment on milk proteins and lipids. J. Agric. Food Chem. 2008, 56, 1984-1991. [CrossRef]

137. Fernández, M.; Ganan, M.; Guerra, C.; Hierro, E. Protein oxidation in processed cheese slices treated with pulsed light technology. Food Chem. 2014, 159, 388-390. [CrossRef]

138. Cantos, E.; Espín, J.C.; Tomás-Barberán, F.A. Postharvest induction modeling method using UV irradiation pulses for obtaining resveratrol-enriched table grapes: A new "functional" fruit? J. Agric. Food Chem. 2001, 49, 5052-5058. [CrossRef]

139. Pataro, G.; Sinik, M.; Capitoli, M.M.; Donsì, G.; Ferrari, G. The influence of post-harvest UV-C and pulsed light treatments on quality and antioxidant properties of tomato fruits during storage. Innov. Food Sci. Emerg. Technol. 2015, 30, 103-111. [CrossRef]

140. Braga, T.R.; Silva, E.O.; Rodrigues, S.; Fernandes, F.A. Drying of mangoes (Mangifera indica L.) applying pulsed UV light as pretreatment. Food Bioprod. Process. 2019, 114, 95-102. [CrossRef]

141. Wiktor, A.; Mandal, R.; Singh, A.; Pratap Singh, A. Pulsed Light treatment below a Critical Fluence $\left(3.82 \mathrm{~J} / \mathrm{cm}^{2}\right)$ minimizes photo-degradation and browning of a model Phenolic (Gallic Acid) Solution. Foods 2019, 8, 380. [CrossRef] 
142. Chung, S.Y.; Yang, W.; Krishnamurthy, K. Effects of pulsed UV-light on peanut allergens in extracts and liquid peanut butter. J. Food Sci. 2008, 73, 400-404. [CrossRef]

143. Meinlschmidt, P.; Ueberham, E.; Lehmann, J.; Reineke, K.; Schlüter, O.; Schweiggert-Weisz, U.; Eisner, P. The effects of pulsed ultraviolet light, cold atmospheric pressure plasma, and gamma-irradiation on the immunoreactivity of soy protein isolate. Innov. Food Sci. Emerg. Technol. 2016, 38, 374-383. [CrossRef]

144. Yang, W.W.; Chung, S.-Y.; Ajayi, O.; Krishnamurthy, K.; Konan, K.; Goodrich-Schneider, R. Use of Pulsed Ultraviolet Light to Reduce the Allergenic Potency of Soybean Extracts. Int. J. Food Eng. 2010, 6, 1-12. [CrossRef]

145. Shriver, S.; Yang, W.; Chung, S.Y.; Percival, S. Pulsed ultraviolet light reduces immunoglobulin E binding to Atlantic white shrimp (Litopenaeus setiferus) extract. Int. J. Environ. Res. Public Health 2011, 8, 2569-2583. [CrossRef] [PubMed]

146. Li, Y.; Yang, W.; Chung, S.Y.; Chen, H.; Ye, M.; Teixeira, A.A.; Gregory, J.F.; Welt, B.A.; Shriver, S. Effect of pulsed ultraviolet light and high hydrostatic pressure on the antigenicity of almond protein extracts. Food Bioprocess Tech. 2013, 6, 431-440. [CrossRef]

147. Panozzo, A.; Manzocco, L.; Lippe, G.; Nicoli, M.C. Effect of pulsed light on structure and immunoreactivity of gluten. Food Chem. 2016, 194, 366-372. [CrossRef]

148. Orcajo, J.; Lavilla, M.; Martínez-de-Marañón, I. Effect of Pulsed Light treatment on $\beta$-lactoglobulin immunoreactivity. LWT Food Sci. Technol. 2019, 112, 108231. [CrossRef]

149. Moreau, M.; Lescure, G.; Agoulon, A.; Svinareff, P.; Orange, N.; Feuilloley, M. Application of the pulsed light technology to mycotoxin degradation and inactivation. J. Appl. Toxicol. 2013, 33, 357-363. [CrossRef]

150. Funes, G.J.; Gómez, P.L.; Resnik, S.L.; Alzamora, S.M. Application of pulsed light to patulin reduction in McIlvaine buffer and apple products. Food Control 2013, 30, 405-410. [CrossRef]

151. Wang, B.; Mahoney, N.E.; Pan, Z.; Khir, R.; Wu, B.; Ma, H.; Zhao, L. Effectiveness of pulsed light treatment for degradation and detoxification of aflatoxin B 1 and B 2 in rough rice and rice bran. Food Control 2016, 59, 461-467. [CrossRef]

152. Chen, D.; Chen, P.; Cheng, Y.; Peng, P.; Liu, J.; Ma, Y.; Liu, Y.; Ruan, R. Deoxynivalenol decontamination in raw and germinating barley treated by plasma-activated water and intense pulsed light. Food Bioprocess Tech. 2019, 12, 246-254. [CrossRef]

153. Pellicer, J.A.; Navarro, P.; Gómez-López, V.M. Pulsed light inactivation of polygalacturonase. Food Chem. 2019, 271, 109-113. [CrossRef]

154. Jeon, M.S.; Park, K.M.; Yu, H.; Park, J.Y.; Chang, P.S. Effect of intense pulsed light on the deactivation of lipase: Enzyme-deactivation kinetics and tertiary structural changes by fragmentation. Enzyme Microb. Technol. 2019, 124, 63-69. [CrossRef] [PubMed]

155. Teichmann, A.; Dutta, P.C.; Staffas, A.; Jägerstad, M. Sterol and vitamin D2 concentrations in cultivated and wild grown mushrooms: Effects of UV irradiation. LWT Food Sci. Techol. 2007, 40, 815-822. [CrossRef]

156. Koyyalamudi, S.R.; Jeong, S.C.; Pang, G.; Teal, A.; Biggs, T. Concentration of vitamin D2 in white button mushrooms (Agaricus bisporus) exposed to pulsed UV light. J. Food Compost. Anal. 2011, 24, 976-979. [CrossRef]

157. Kalaras, M.D.; Beelman, R.B. Vitamin D2 Enrichment in Fresh Mushrooms Using Pulsed UV Light. Foodscience. 2008. Available online: https://foodscience.psu.edu/directory/rbb6/VitaminDEnrichment.pdf (accessed on 10 October 2019).

158. Kalaras, M.D.; Beelman, R.B.; Elias, R.J. Effects of Postharvest Pulsed UV Light Treatment of White Button Mushrooms (Agaricus bisporus) on Vitamin D2 Content and Quality Attributes. J. Agric. Food Chem. 2011, 60, 220-225. [CrossRef] [PubMed]

159. Chen, S.Y.; Huang, S.J.; Cheng, M.C.; Chen, Y.K.; Yang, S.C.; Mau, J.L. Enhancement of vitamin D2 content in Pleurotus mushrooms using pulsed light. J. Food Process. Pres. 2015, 39, 2027-2034. [CrossRef]

160. Chalupa, W.F.; Schroeder, G.M. Method and Apparatus for Vitamin D Enhancement in Dried Mushroom Powder. U.S. Patent Application No. 13/469,168, 4 September 2014.

161. Schroeder, G.M. Method and Apparatus for Vitamin D Enhancement in Mushrooms. U.S. Patent No. 8,545,915, 1 October 2013.

(C) 2020 by the authors. Licensee MDPI, Basel, Switzerland. This article is an open access article distributed under the terms and conditions of the Creative Commons Attribution (CC BY) license (http://creativecommons.org/licenses/by/4.0/). 